\title{
Monomeric $\alpha$-Synuclein activates the Plasma Membrane Calcium Pump
}

Antoni Kowalski ${ }^{1,3,4}$, Cristine Betzer ${ }^{2,3}$, Sigrid Thirup Larsen ${ }^{1,3}$, Emil Gregersen ${ }^{2,3}$, Montaña Caballero Bermejo ${ }^{1,3,5}$, Poul Henning Jensen ${ }^{2,3}$, Poul Nissen ${ }^{1,3}$

${ }^{1}$ Department of Molecular Biology and Genetics, Aarhus University, Aarhus, Denmark

${ }^{2}$ Department of Biomedicine, Aarhus University, Aarhus, Denmark

${ }^{3}$ Danish Research Institute of Translational Neuroscience - DANDRITE, Aarhus University, Aarhus, Denmark

${ }^{4}$ Department of Drug Design and Pharmacology, University of Copenhagen, Denmark

${ }^{5}$ Department Biochemistry and Molecular Biology and Genetics, IBMP, University of Extremadura, Badajoz, Spain

correspondence: antoni.kowalski@dandrite.au.dk; phj@mbg.au.dk and pn@mbg.au.dk

\begin{abstract}
Alpha-synuclein (aSN) is a membrane-associated and intrinsically disordered protein, wellknown for pathological aggregation in neurodegeneration. The physiological function of aSN however is disputed. Pull-down experiments have pointed to plasma membrane $\mathrm{Ca}^{2+}$-ATPase (PMCA) as a potential interaction partner. From proximity ligation assays we find here that aSN and PMCA colocalize at neuronal synapses, and that calcium expulsion is activated by aSN and PMCA. From PMCA activity studies we show that soluble, monomeric aSN activates PMCA at par with calmodulin, yet independent of the autoinhibitory domain of PMCA, but highly dependent on acidic phospholipids and the membrane-anchoring N-terminus of aSN. On the PMCA molecule, the interaction site is mapped to the acidic lipid-binding site, located within a PMCA-specific linker region connecting the cytosolic A domain and transmembrane segment 3. Our studies point towards a physiological role of monomeric aSN as a stimulator of calcium clearance in neurons through activation of PMCA.
\end{abstract}

\section{INTRODUCTION}

Alpha-synuclein (aSN, 140 residues, and $14.5 \mathrm{kDa}$ molecular weight) is an intrinsically disordered protein and is well recognized and highly studied for its pathological role in neurodegeneration. Oligomerization, fibrillization, and abnormal aggregation in neurons are 
linked to synucleinopathies, e.g., Lewy body dementia or Parkinson's disease (PD). However, the normal function of monomeric aSN remains an open question. Importantly, aSN is highly abundant in the presynaptic region of neurons, where concentrations reach 5-50 $\mu \mathrm{M}$ (Bodner et al., 2009; Perni et al., 2017; Theillet et al., 2016). Even at a level this high the protein exists in cells as a structurally disordered monomer (Fauvet et al., 2012; Theillet et al., 2016). Alphasynuclein is also a peripheral membrane protein by interactions of a partially helical structure in the positively charged N-terminal region 1-95 with phospholipids (Dikiy and Eliezer, 2012). The ability of aSN to interact with acidic phospholipids is important for such functions as clustering of synaptic vesicles (Jo et al., 2000; Lautenschlager et al., 2018), regulation of the presynapse size (Vargas et al., 2017), and neurotransmitter release by promoting the formation of SNARE complexes (Burre et al., 2010). Disease-related mutation A30P - strongly linked to the inherited form of PD - result in diminished lipid-binding properties (Jensen et al., 1998). The C-terminal region, comprising residues 96-140 remains unfolded regardless of interactions, has a strong negative charge, and binds calcium ions with low affinity (Eliezer et al., 2001; Lautenschlager et al., 2018; Nielsen et al., 2001).

Regulation of intracellular calcium homeostasis is essential for the proper functioning of cells. Resting concentration in cytosol of a healthy cell is low, around $100 \mathrm{nM}$, while the extracellular concentration is in the millimolar range. In a calcium signaling event, rapid calcium influxes via calcium channels must be followed by efficient recovery by specialized proteins. The key players are i) the plasma membrane calcium ATPase (PMCA) and ii) the sodium-calcium exchanger (NCX) that are both removing $\mathrm{Ca}^{2+}$ to the extracellular space, and iii) SERCA filling intracellular calcium stores. Furthermore, calcium-binding proteins, in particular calmodulin (CaM), function as a calcium buffer and calcium-dependent regulator of multiple proteins (Cali et al., 2018).

Increasing evidence reveals a link between calcium dysregulation and propagation of synucleinopathies. Voltage-gated Cav1 channels and Cav1.3 mRNA are upregulated as an early feature of PD in areas not associated with overt loss of neurons or Lewy body formation (Hurley et al., 2013; Hurley et al., 2015). Fibrillar oligomers of aSN, which are formed with the disease development, can enhance the $\mathrm{Ca}^{2+}$-permeability of plasma membrane (Cali et al., 2014; Di Scala et al., 2016; Rcom-H'cheo-Gauthier et al., 2016) and have been found to activate the sarco/endoplasmic reticulum $\mathrm{Ca}^{2+}$-ATPase (SERCA) (Betzer et al., 2018), thus contributing to disturbances in calcium homeostasis. The SERCA interaction was identified by aSN pull-down experiments, and PMCA was also identified from these experiments (Betzer et al., 2015). 
Neurons have a large complexity and a highly polarized architecture; calcium signaling events in these cells, therefore, are highly localized. Dramatic, but extremely local calcium influxes are related to signals leading to vesicular exocytosis in the presynaptic termini. In the presynaptic bouton, the signaling events take place in nanodomains within $50 \mathrm{~nm}$ from $\mathrm{Ca}^{2+}$ channels (Augustine et al., 2003) and free calcium concentrations can rise locally by $>1000$ fold (Long et al., 2008). Terminating, rather than dissipating a local signal causes a high demand for an efficient and flexible calcium removal system with fine-tuning to a required level of resting concentrations.

PMCA is a single polypeptide transmembrane protein of $130-140 \mathrm{kDa}$ and belongs to the P2B subfamily of P-type ATPases. Being a high affinity and low-capacity active transporter, it can fine-tune the resting free calcium ion concentration in cytosol (Carafoli, 1994; Strehler et al., 2007b). In humans and other mammals, four PMCA isoforms (PMCA1-4) are encoded by separate genes (Strehler et al., 2007a). PMCA1 is considered a "housekeeping" pump and together with PMCA4 ubiquitously expressed in all tissues. PMCA2 and 3 have specific expression patterns and are mostly found in excitable tissues and often referred to as neuronspecific isoforms. (Domi et al., 2007; Strehler and Thayer, 2018). Through alternative RNA splicing at two different sites ("A" and "C"), the four isoforms can be produced in more than 20 different variants (Strehler and Zacharias, 2001). Splicing at site $\mathrm{C}$ affects the length of the C-terminal tail with calmodulin sites and splicing at site A impacts the length of the first intracellular loop which leads from the A-domain to the transmembrane segment 3 (TM3) (Strehler, 2015). The different splice variants are in many cases tissue-specific and differ in the degree of activation and autoinhibition (Caride et al., 1999; Kessler et al., 1992).

PMCA is regulated through interactions with protein partners as well as phospholipids. A classical regulatory feedback mechanism happens via the C-terminal autoinhibitory domain of PMCA. In the resting cell, the domain interacts with the cytoplasmic domains and autoinhibits calcium transport. Upon an increase in intracellular calcium, calcium-bound calmodulin $(\mathrm{CaM})$ binds to the autoinhibitory domain resulting in a rise in PMCA activity. The degree and calcium threshold of activation may depend on how many CaM-binding sites - one or two - are present within the autoinhibitory domain (Tidow et al., 2012). Furthermore, PMCA regulation by acidic phospholipids has been mapped to two binding sites - one in the autoinhibitory domain, the other at the cytosolic loop between the A-domain and TM3. The mechanism of how acidic lipids regulate the pump is not understood in detail; however, they appear to be both modulators of activation by $\mathrm{CaM}$ as well as stand-alone activators (Brodin et al., 1992; Lopreiato et al., 2014; Niggli et al., 1981a; Penniston et al., 2014; Pinto Fde and Adamo, 2002; Tidow et al., 
2012; Zvaritch et al., 1990). Hence, the autoregulatory regions also correspond to the sites of variations by alternative splicing.

In the present study, we show that aSN in its soluble, monomeric form acts as a very potent activator of human PMCAs. The effect relies on the presence of acidic phospholipids and is independent of the calmodulin-binding autoinhibitory domain. Our findings suggest that the activation mechanism is based on interactions of the $\mathrm{N}$-terminal segment of aSN with the acidic phospholipid binding site of PMCAs. We propose that aSN complements calmodulin in local compartments such as the presynapse. Our finding gives a new perspective on the physiological role of native aSN in the presynapse and demonstrates how the lipid environment affects the critical calcium extruding activity of PMCA.

\section{RESULTS}

\section{PMCAs are interaction partners for aSN}

In previous mass spectrometry studies, PMCA was noted as a potential interaction partner of aSN (Betzer et al., 2015). Further co-immunoprecipitation experiments analyzed by western blotting show that endogenous PMCA interacts with endogenous aSN (Figure 1A, right). Coimmunoprecipitation experiments with aSN-knockout brain homogenate supplemented with either purified aSN monomer, or in vitro formed aSN oligomer revealed that PMCA did not display preferential aSN-conformational binding as interactions occur with both the monomer and the oligomer (Figure 1A, left).

The interaction between aSN and PMCA was investigated further in primary hippocampal neurons isolated from newborn C57B1/6 mice. Primary hippocampal neurons were analyzed after 14 days in culture by immunofluorescence labeling of synapses by synaptophysin and an aSN/PMCA proximity ligation assay (PLA) yielding a red fluorescent signal when aSN and PMCA are located within 40nm. PMCA and aSN were found to be in this proximity of each other and located to the synapses (Figure 1B).

The functional effect of PMCA calcium transport was investigated in primary hippocampal neurons from aSN-KO neurons transiently transfected with either mCherry or aSN and mCherry. The sarco-/endoplasmic reticulum $\mathrm{Ca}^{2+}$-ATPase (SERCA) was inhibited by thapsigargin $(4 \mu \mathrm{M})$ before the recording. Cellular calcium responses were monitored by the calcium-sensing dye, Fura2-AM upon depolarization by $8 \mathrm{mM} \mathrm{KCl}$ in the extracellular medium. Neurons expressing aSN expel calcium to a markedly higher degree than the mCherry expressing neurons (Figure 1C). The calcium expulsion was Ca2+-ATPase dependent as the 
inhibition by vanadate decreased the calcium expulsion in both the mCherry expressing neurons and the aSN mCherry neurons.

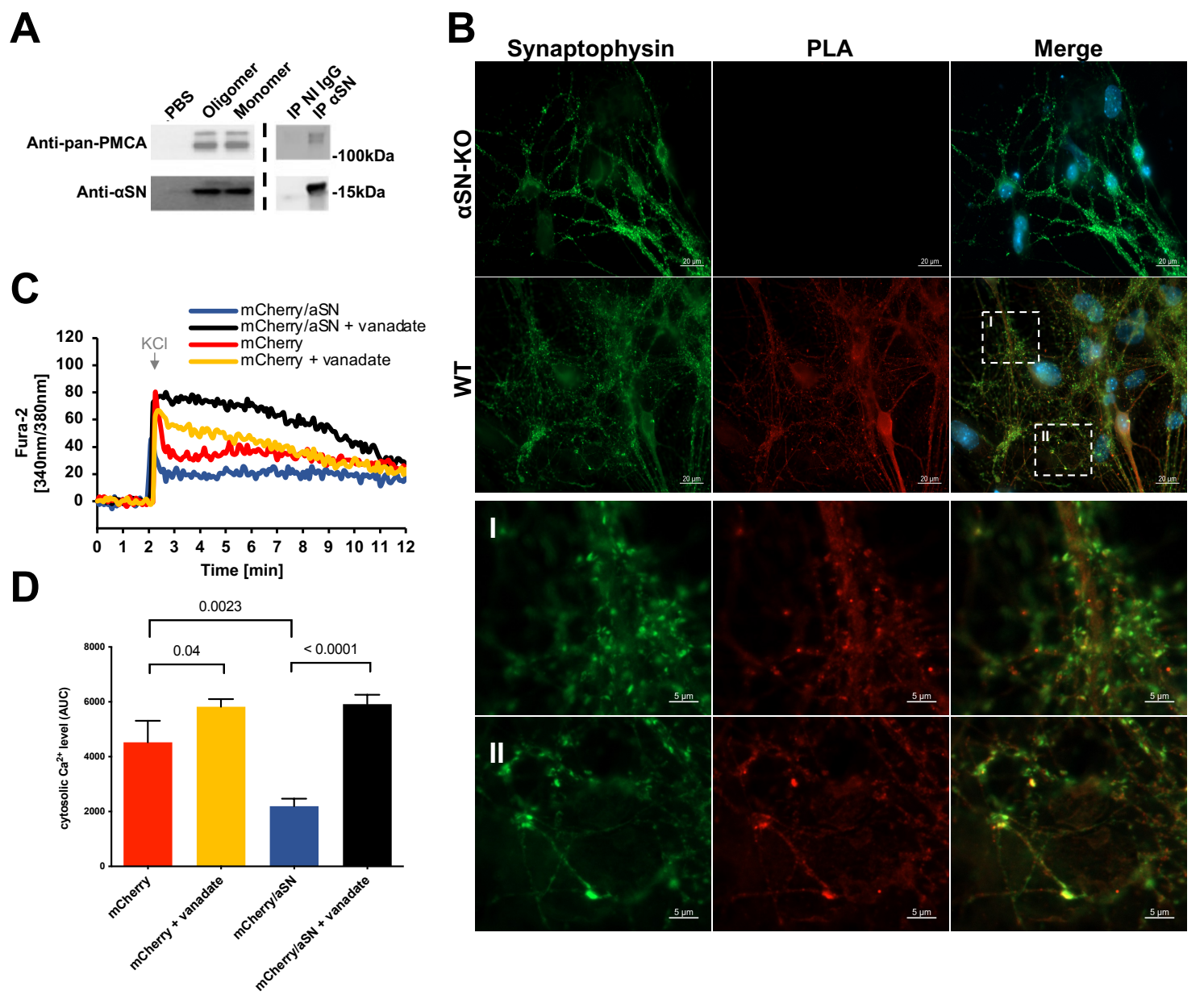

Figure 1. aSN is an interaction partner to PMCA and stimulates calcium extrusion.

A. Co-immunoprecipitation of PMCA with aSN.

Left: In detergent extract of total brain homogenates from aSN-knockout (aSN-KO) mice both exogenous aSN monomer and in vitro formed oligomers are pulled down together with PMCA mice by aSN binding sepharose (ASY-1). Control without exogeneous aSN (PBS) confirms no unspecific antibody binding. Right: In the detergent extract of total brain homogenate from C57BL/ 6 mice endogenous aSN is pulled down together with the endogenous PMCA by aSN specific antibody (ASY-1) and not by control non-immune antibody (NI IgG).

B. Proximity ligation assay (PLA) of aSN and PMCA primary hippocampal neurons

Proximity ligation assay (PLA) of PMCA and aSN in primary hippocampal neurons made from WT or aSNKO mice. Left column: synapses visualized by synaptophysin labeling. Middle column: red fluorescence signal represents positive PLA result, meaning proximity of aSN and PMCA of less than $40 \mathrm{~nm}$. Right column: Merged PLA and synaptophysin images show PMCA and aSN interaction localized to the synapses. I and II: Zoomed-in images of boxed areas of WT primary hippocampal neurons.

$C$ and D. aSN increases calcium export from depolarized primary neurons.

Cytosolic calcium was monitored by Fura-2-AM loaded into DIV8 neurons. Before recording SERCA was inhibited by thapsigargin. Calcium influx was induced by the addition of $\mathrm{KCl}$. At the recording time of 2 $\min . \mathrm{KCl}$ was added to depolarize the neurons and the calcium response was followed over time.

C - Representative curves from measurements in: blue - neurons expressing mCherry and aSN, black: neurons expressing mCherry and aSN treated with $1 \mu \mathrm{M}$ vanadate to inhibit ATPases, red - neurons expressing mCherry alone, yellow - neurons expressing mCherry alone and treated with $1 \mu \mathrm{M}$ vanadate. 
D - Cytosolic $\mathrm{Ca}^{2+}$ level after the KCl-induced influx, quantified as the area under the curve (AUC $\pm \mathrm{SEM}$ ). The response upon $\mathrm{K}^{+}$induced depolarization was quantified as the Area Under Curve (AUC) from each measured neuron in the 2-12 min. interval.

$\mathrm{N}(\mathrm{mCherry} / \mathrm{aSN})=7, \mathrm{~N}($ mCherry/aSN + vanadate $)=10, \mathrm{~N}($ mCherry $)=6$, and $\mathrm{N}($ mCherry + vanadate $)$ $=10$. The colors of the bars correspond to the top figure. Data presented as mean \pm SEM. Statistical analysis is conducted as multiple comparisons with one-way ANOVA combined with Sidak post hoc test.

\section{Monomeric alpha-synuclein activates human PMCA in a lipid-dependent manner and independent of the autoinhibitory domain of PMCA}

The measurements of calcium-dependent ATPase activity of PMCA were performed with two available human PMCA variants for which expression and purification had been established in the laboratory - ubiquitous PMCA1 and neuron-specific PMCA2 isoforms (specifically PMCA1d and PMCA2w/a splice variants). The experiments were performed with PMCAs relipidated either with porcine brain phosphatidylcholine (BPC, neutral lipids) or with bovine brain lipid extract Folch fraction I (from here on referred to as brain extract, BE), $60 \%$ of which consists of negatively charged phosphatidylinositol and phosphatidylserine lipids. We observed monomeric aSN causing a strong increase in activity of both PMCA isoforms, however only for PMCA relipidated with $\mathrm{BE}$, not $\mathrm{BPC}$. As the titration of free $\mathrm{Ca}^{2+}$ reveals, the elevation of ATPase activity is accompanied by a great rise in the apparent calcium affinity (Figure 2A, left, center; Table 1). Additionally, the activation is gradually abolished by increasing the content of neutral BPC in the relipidation mixture (Figure 2A, right). To rule out potential, unspecific effects of other ATPases or impurities in the PMCA preparations, we prepared an inactive mutant of PMCA (PMCA1 $\left.\mathrm{d}_{\mathrm{D} 475 \mathrm{~N}}\right)$, in which the reactive aspartyl residue in the active site was replaced by asparagine. In terms of purity, these preparations were no different from the wild type, as confirmed by SDS-PAGE and size exclusion chromatography (Figure S1), and they showed no ATPase activity, neither with nor without aSN (Figure 2A left, 2B).

To further investigate the acidic phospholipid dependence, we performed aSN titration comparing effects of wild type aSN and its A30P mutant with reduced membrane-binding ability (Jensen et al., 1998). In BE, the activation of PMCA was significantly attenuated by A30P mutation. As could be expected none of the aSN variants were effective in the neutral environment of BPC (Figure 2B).

Next, we examined the potential interplay between aSN and the best-known protein activator of PMCA - calmodulin. In both PMCA isoforms, the susceptibility for activation by $\mathrm{CaM}$ and aSN appear to be lipid-dependent, with the acidic lipids promoting the effect of aSN, and neutral lipids promoting the effect of CaM (Figure 2C). To confirm that the action of aSN 
was independent of the autoinhibitory domain, we designed a truncated construct (PMCA1$\Delta \mathrm{C}$ ), lacking 184 residues of the C-terminus including the autoinhibitory domain with the calmodulin-binding site and a long, intrinsically disordered linker region (Figure 2D, top) Foreseeably, loss of the C-terminal tail resulted in a constitutively active protein, insensitive to calmodulin (Figure S3) with basal activity higher than that of the full-length wild type (Figure 2D, bottom left). However, the stimulation by aSN was preserved and appeared like in the fulllength pump, indicating the aSN interaction is independent of the autoinhibitory mechanism (Figure 2D, bottom right). The apparent $\mathrm{Ca}^{2+}$ affinity of PMCA1d was increased by the truncation itself, and aSN strengthened that effect (Table 1). Again, the activation was lipiddependent, occurring in PMCA1- $\Delta \mathrm{C}$ constructs reconstituted in BE and not in BPC (Figure S3).

Additionally, we examined the oligomeric form of the full-length aSN, which was previously established to have a great activating impact on the SERCA (Betzer et al., 2018). For PMCA, however, the effect was smaller than that of the monomer, occurring only at higher concentrations and to a lower fold of activation (Figure S3D). The activation might simply be explained by dissociating monomer, which we have found to be present in oligomer preparations (Figure S2). 
A

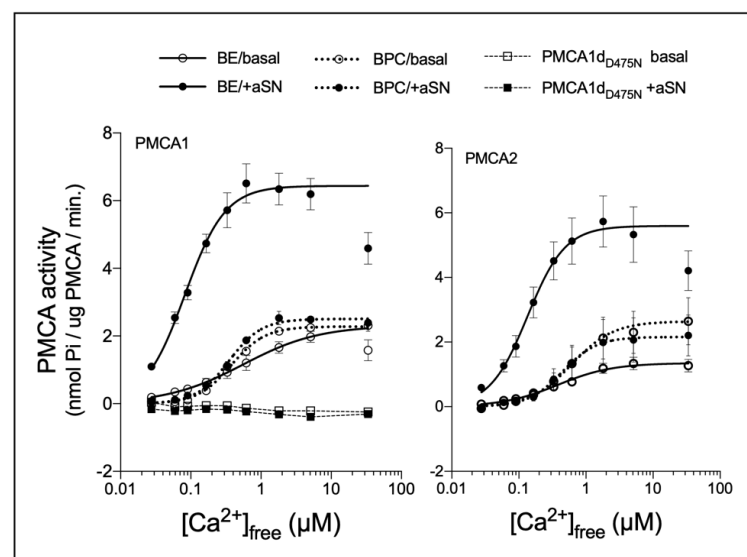

C

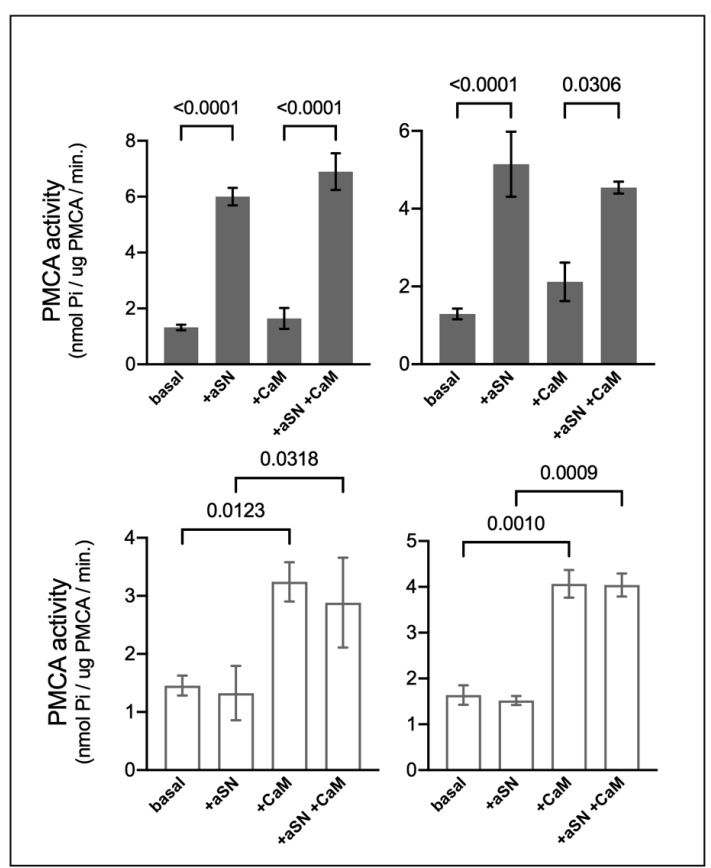

B

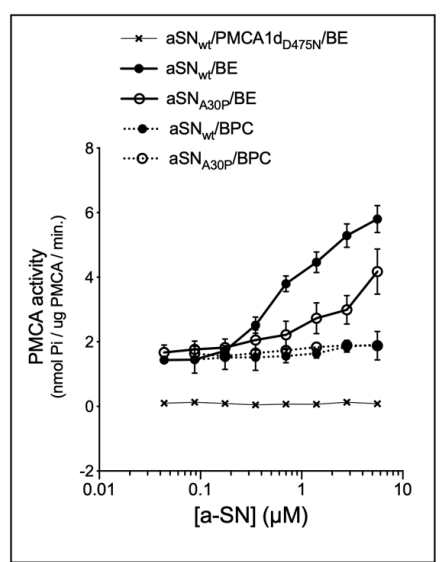

D


Figure 2. Monomeric alpha-synuclein activates human PMCA in a lipid-dependent and autoinhibitory domain-independent manner

A. Alpha-synuclein stimulates plasma membrane calcium ATPase activity in an acidic, but not neutral lipid environment.

aSN activates PMCA1d (left) and PMCA2w/a (center) in the presence of brain extract (BE, solid line), but not in presence of brain PC (BPC, dotted line). PMCA activity was measured as a function of free $\mathrm{Ca}^{2+}$ concentration $\left(\left[\mathrm{Ca}^{2+}\right]\right.$ free $)$. Empty symbols represent basal activity and filled symbols the activity in presence of $2.8 \mu \mathrm{M}$ aSN. The lines are the best fit given by the Hill equation with $\mathrm{Kd}$ values listed in Table 1. To rule out nonspecificity of ATP hydrolysis observed in wild-type PMCA, an inactive D475N mutant of PMCA1d was tested (squares, dashed line), Right: Fold activation of PMCAs by aSN decreases with the increasing amount of neutral lipids. Brain PC was titrated in brain extract the relipidation of PMCA. Empty symbols - PMCA1d, filled symbols - PMCA2w/a. Data in the calcium titration experiment are mean \pm s.e.m., for some points, the error bars are smaller than symbols. PC titration data are from a single experiment for each of the PMCA isoforms.

B. Compared to wild-type, A30P a-SN mutant has reduced ability to activate PMCA in presence of acidic lipids.

PMCA1d activity was measured as a function of a-SN (wild-type or A30P) concentration in presence of $1.8 \mu \mathrm{M}$ free $\mathrm{Ca}^{2+}$. PMCA1d was relipidated either with brain extract (BE) or brain phosphatidylcholine (BPC). Neither of the aSN variants activated PMCA in a neutral lipid environment. Simultaneously we experimented with an inactive mutant of PMCA1d (D475N), which was not stimulated by a-SN in presence of BLE. Data are mean \pm s.e.m. For some of the points, the error bars are smaller than the symbols. 
C. Neutral or acidic lipids create a condition for PMCA being activated exclusively by calmodulin or a-SN. Bars display the PMCA1d (left) and PMCA2w/a (right) activity without activator, with a-SN, with calmodulin $(\mathrm{CaM})$, and both activators. PMCA was relipidated in BE (top, filled bars) or brain BPC (bottom, empty bars).

D. The activation by ASN is independent of the PMCA's autoinhibitory domain. Top: Comparison of two PMCA1 constructs - full-length PMCA1d with calmodulin-binding sites and PMCA1- $\Delta \mathrm{C}$, where C-terminal tail, containing the autoinhibitory domain with calmodulin-binding sites, was removed by cleavage with TEV protease. TEV proteolytic cleavage site was introduced by site-directed mutagenesis at residue 1074. Bottom left: Schematic representation of PMCA1d with TEV cleavage site introduced by site-directed mutagenesis. Middle right: Basal activity of PMCA1d and PMCA1- $\triangle \mathrm{C}$ in presence of either acidic lipids or PC. Bottom right: The activity of PMCA1- $\Delta \mathrm{C}$ with (filled symbols) and without $2.8 \mu \mathrm{M}$ aSN (empty symbols) as a function of increasing $\left[\mathrm{Ca}^{2+}\right] \mathrm{free}_{\text {. }}$ PMCA1- $\Delta$ C was relipidated with BE. The lines are the best fit given by the Hill equation with Kd values listed in Table 1.

Except for cases of $\mathrm{Ca}^{2+}$ or aSN titration, the fixed concentrations were $1.8 \mu \mathrm{M}$ or $5.6 \mu \mathrm{M}$, respectively. Relevant no-calcium or no-aSN backgrounds were subtracted. Data from at least three independent experiments are expressed as mean \pm s.e.m. Statistical analysis was conducted in GraphPad Prism software as multiple comparisons with one-way ANOVA combined with Sidak post hoc test.

Subfigure 2B (bottom left) as well as 3C, 3D (bottom right), 4A, 4C and 4E were created with Biorender.com

\begin{tabular}{|c|c|c|c|c|}
\hline & BE -aSN & BE $+\mathbf{a S N}$ & BPC -aSN & BPC + aSN \\
\hline PMCA1d & $0.55 \pm 0.019$ & $0.08 \pm 0.004$ & $0.38 \pm 0.016$ & $0.34 \pm 0.019$ \\
\hline PMCA1- $\Delta C$ & $0.16 \pm 0.01$ & $0.06 \pm 0.006$ & $0.12 \pm 0.008$ & $0.10 \pm 0.010$ \\
\hline PMCA2w/a & $0.38 \pm 0.047$ & $0.12 \pm 0.02$ & $0.57 \pm 0.043$ & $0.44 \pm 0.021$ \\
\hline
\end{tabular}

Table 1. $\mathrm{K}_{\mathrm{d}}\left(\mathrm{Ca}^{2+}\right)$ values $(\mu \mathrm{M})$ of PMCAs in different lipid environments. BE - brain lipid extract containing negatively charged phosphatidylinositol (PI) and phosphatidylserine (PS); BPC - brain phosphatidylcholine (PC). Values calculated in GraphPad Prism from data presented in Figures 2A and D.

\section{PMCA alternative splicing events correlate with a-SN expression level}

PMCA is extensively regulated by alternative splicing, which results in over 20 variants, differing in distribution, kinetic properties, and sensitivity to interaction partners e.g., calmodulin (Krebs, 2015; Strehler and Zacharias, 2001). We took into consideration that some of them could vary also in how they respond to aSN. We used available transcriptomics data from the VastDB database (http://vastdb.crg.eu/), containing information on alternative splicing events. The level of alternative splicing events in different tissues are indicated by PSI values ("percent spliced in"), and we asked whether specific splicing events correlate with aSN expression levels. First, we analyzed the splice site $\mathrm{C}$, which is located at the $\mathrm{C}$-terminal region of PMCA and contains the autoinhibitory domain that interacts with CaM. Alternative splicing at this site gives variants that may differ in the degree of autoinhibition or rate of activation by CaM. The splice variant "a", with a shortened autoinhibitory domain is known to have higher basal activity and to be less sensitive to CaM stimulation (Caride et al., 2007). The available data regarded PMCA1, PMCA3, and PMCA4, and the analysis displayed in Figure 3A shows that in the case of PMCA1 and PMCA4 exon incorporation leading to the variant "a" positively 
correlate with expression level of aSN with only a few outliers. Only PMCA3 did not correlate and the PSI value of the "a" variant was relatively high regardless of aSN expression interval. The results suggest that in tissues highly expressing aSN, PMCA is alternatively spliced in a way that favors less CaM-dependent variants. The PMCA "a" variant is mainly expressed in brain tissues and explains the positive correlation between PMCA and aSN. Except for brain tissues aSN is also expressed in high amounts in melanocytes and bone marrow which account for the two outliers in the plot.

The other of the two splice sites - A - is located at the lengthy A-TM3 linker region between the A-domain and TM3. Among isoforms, PMCA1 is not being spliced in this region, while in PMCA2 alternative exon composition leads to 4 different variants named " $z$ ', " $x$ ", " $y$ ", and "w", with all of them except "y" having been detected in humans (Di Leva et al., 2008; Strehler et al., 2007b). Figure 3B displays the analysis of the PMCA2 splicing events, where three exons can be incorporated leading to variants "w" (full length containing sequences marked in orange, yellow and blue) and " $x$ " (containing the blue sequence). The " $z$ " variant occurs when none of the three exons are incorporated (Figure 3C). We observed incorporation of the exons leading to variants with a longer A domain linker insert in tissues with low aSN expression and barely any incorporation of these exons for tissue with high expression of aSN. This suggests that low expressing tissues would preferentially transcribe the longest PMCA2w, and tissues richer in aSN would express more PMCA2x and $\mathrm{z}$.

The extended, variable A-TM3 linker of PMCA is predicted to adopt a loop structure with a high level of disorder (Figure 3D), and near TM3 it contains several positively charged residues that mediate the activation by acidic phospholipids (Brini et al., 2010; Brodin et al., 1992; Pinto Fde and Adamo, 2002). We speculated about the importance of the linker length in the interaction with disordered aSN and performed the free calcium titration experiment with PMCA2 variants w/a, x/a, and z/a. All PMCA2 constructs used in the experiment were transformed anew into yeast cells derived from one colony and were expressed, purified, and tested simultaneously. The activity assays show that all three variants are activated by aSN (Figure 3E). The most remarkable difference is that PMCA2w/a has the lowest maximum activity among variants when activated by aSN. It was also the only one with a substantial increase in the apparent calcium affinity induced by aSN, the other having high affinity also in the basal activity state. PMCA2z/a, having the shortest linker region, showed the highest basal activity. The results implicate the A-TM3 loop in aSN interactions. 
A

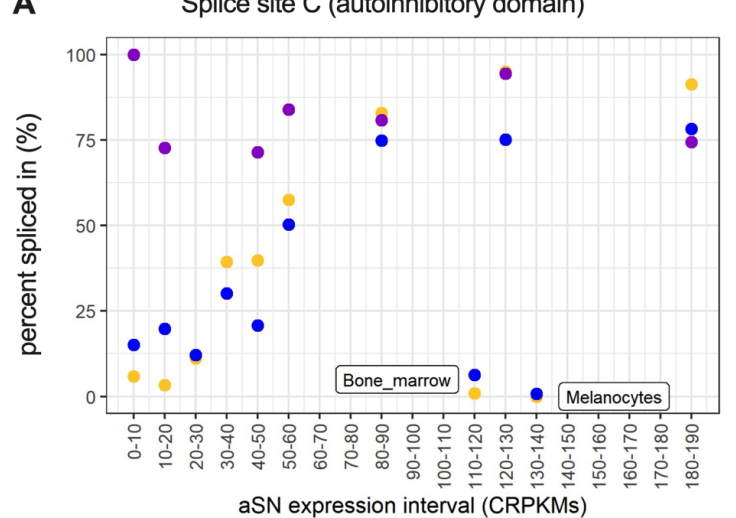

aSN expression interval (CRPKMs)

- PMCA1a - PMCA3a - PMCA4a
B
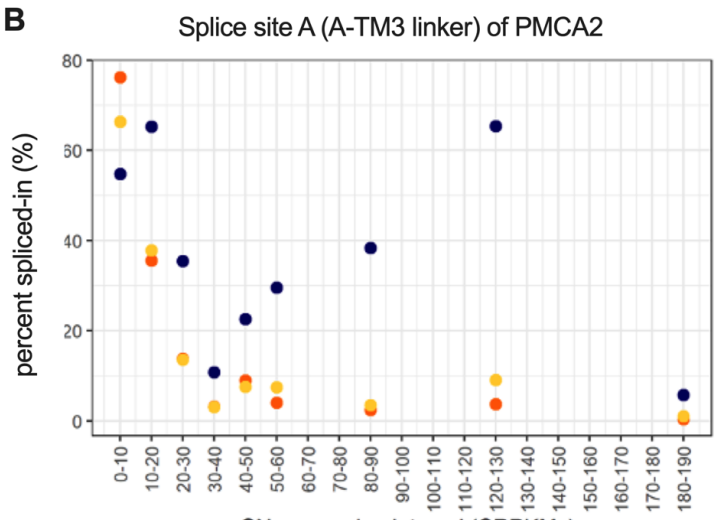

aSN expression interval (CRPKMs)

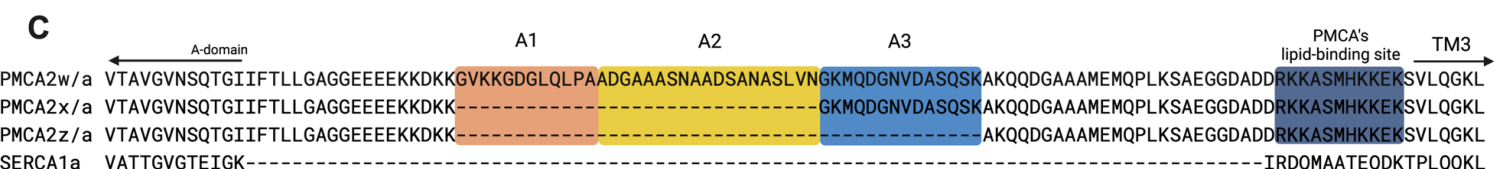

D

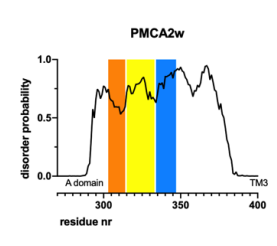

PMCA2z

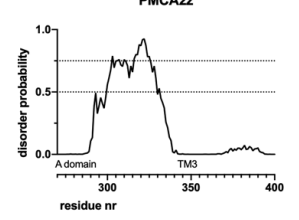

$\mathbf{E}$


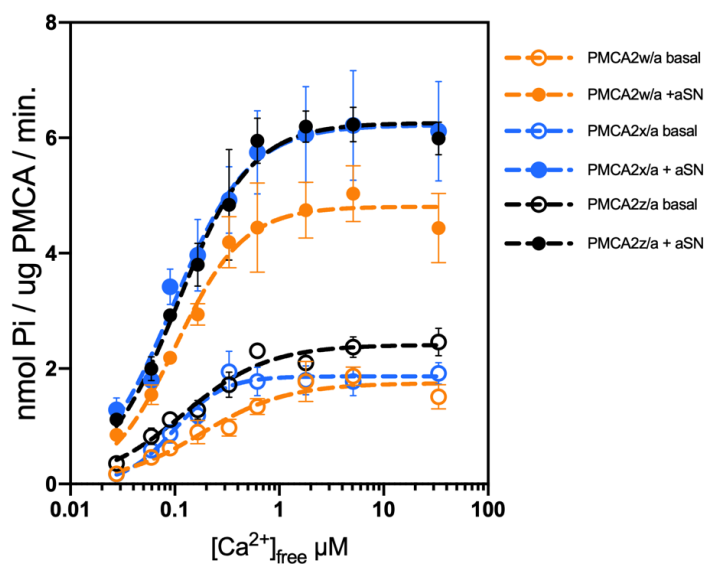

Figure 3. PMCA's alternative splicing events correlate with a-SN expression levels and can modulate activating effect of a-SN

A-B. PMCA's alternative splicing events correlate with a-SN expression levels. The analysis of transcriptomics data from the VastDB database.

A - Exon incorporation for exons at splice site C for PMCA 1,3, and 4. The average value of percent spliced in (PSI) for the exon leading to splice variant "a" as a function of aSN tissue expression.

B - Exon incorporation for exons at splice site A for PMCA 2. The average value of percent spliced in (PSI) for the three possible exons as a function of aSN tissue expression. The color code for the exons (orange, yellow, blue) is consistent with corresponding PMCA regions marked on the subfigures C and D. For VastDB entry numbers of analyzed events refer to the method section

C. Sequence alignment of the extended A-TM3 loop of PMCA2w, $x$, and $\mathbf{z}$ variants. Fragments A1, A2, and A3 of the A-TM3 loop correspond to alternative exons incorporations. Color code corresponds to respective alternative splicing events presented in figure B. Sequence of the corresponding region of SERCA1a for comparison.

D. Disorder prediction of the A-TM3 loop of PMCA2. Top left - PMCA2w, top right-PMCA2x, bottom left - PMCA2z. Bottom right - schematic representation of PMCA2w/a with the intrinsically disordered loop region between domain A and TM3.

E. Comparison of aSN impact on three human splice variants of PMCA2 - calcium dependent activity of PMCA2w, $x$ and $z$. The lines are the best fit given by the Hill equation with the apparent $\mathrm{Kd}$ values $(\mu \mathrm{M})$ for $\mathrm{Ca}^{2+}$ 
as follows: PMCA2w/a basal (without aSN) $-0.178 \pm 0.042$, with aSN $0.102 \pm 0.009$; PMCA2x/a basal -0.098 \pm 0.011 , with aSN $0.097 \pm 0.009 ;$ PMCA2z/a basal $0.127 \pm 0.016$, with aSN $0.106 \pm 0.008$. Values calculated in GraphPad Prism

\section{The aSN-PMCA interaction happens between the $\mathrm{N}$-terminal region of aSN and the acidic phospholipid binding site of PMCA.}

To map the potential interaction site on the aSN sequence (1-140) we performed a comparison of shortened aSN variants, truncated $\mathrm{N}$-terminally $(\Delta 1-14, \Delta 1-29)$ or $\mathrm{C}$-terminally (1-95, 1-61) (Figure 4AB). Most notably, the $\Delta 1-14$ construct had a greatly weakened effect on PMCA and $\Delta$ 1-29 had almost no ability to activate PMCA at all, displaying only minor effects above $5 \mu \mathrm{M}$ concentration. The C-terminally truncated (1-95) construct generally saved its function although to a lesser extent, activating to a lower fold (Figure 4B, Supplementary figure 3B). The (1-61) variant of a-SN did not reach saturation within examined concentration range, which suggests greatly reduced apparent affinity to PMCA.

The disordered C-terminal tail of aSN has calcium-binding properties (Lautenschlager et al., 2018), thus we asked if it would impact the apparent calcium affinity of PMCA, and we compared activation by the full length and C-terminally truncated (1-95) aSN in the calcium titration experiment. However, despite the lower fold-activation, the C-truncated construct did not differ in shifting PMCA's apparent $\mathrm{K}_{\mathrm{d}}$ for $\mathrm{Ca}^{2+}$ (Figure S3B).

Within certain limitations, deletions of large fragments of the lengthy A-TM3 linker of PMCA, including its acidic lipid-binding site, can retain basal activity (Pinto Fde and Adamo, 2002). Guided by this we designed constructs and expressed and purified certain deletion mutants of PMCA2, where we removed large parts of the linker. Sequences of the targeted region PMCA2w/a and the three designed variants $(\Delta 292-383, \Delta 298-383, \Delta 298-372)$ are displayed in Figure 4C. The activity assay in presence of brain lipid extract (Figure 4D) showed differences in the basal activity and response to aSN. We observed a complete loss of ATPase activity of the $\Delta 292-383$ variant. Keeping the six N-terminally located residues of the linker resulted in the $\Delta 298$-383 variant with the basal activity preserved $(0.84 \pm 0.023 \mathrm{nmol}$ $\mathrm{Pi} / \mu \mathrm{g} \mathrm{PMCA} / \mathrm{min}$.), but not responding to aSN titration. Preserving further the 11-residues long binding site for negatively charged lipids at the C-terminal end of the linker, close to TM3 ( $\Delta 298$-372) boosted the basal activity (3,407 $\pm 0,49 \mathrm{nmol} \mathrm{Pi} / \mu \mathrm{g} \mathrm{PMCA} / \mathrm{min}$.) and reinstalled the fold-activation by aSN to the level observed in the wild type PMCA2w/a (but having lower basal activity of $2.33 \pm 0.29 \mathrm{nmol} \mathrm{Pi} / \mu \mathrm{g} \mathrm{PMCA} / \mathrm{min}$ ). As this points to the binding site for negatively charged lipids as crucial for the interaction with aSN, we propose the aSN-PMCA 
interaction mediated by acidic phospholipids happens between the $\mathrm{N}$-terminal region of aSN and the acidic lipid binding site of PMCA (Figure 4E).

A



C
B

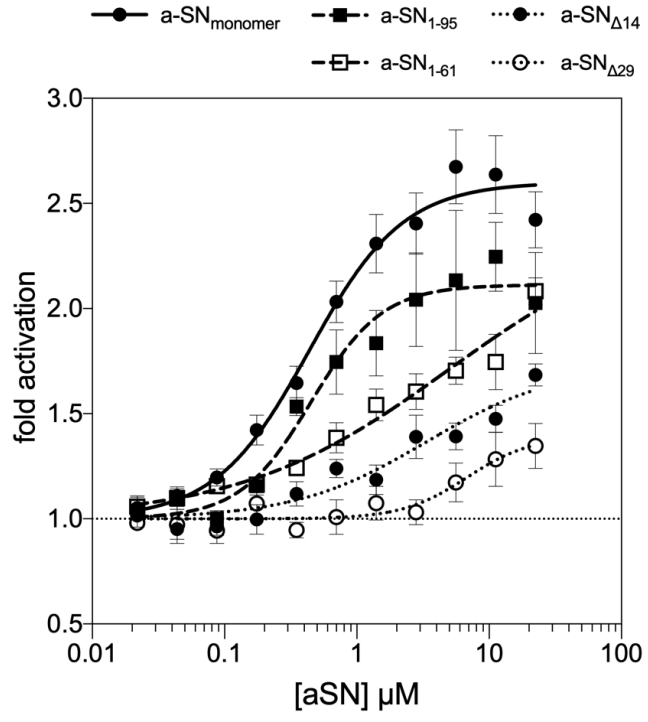





E


Figure 4. The aSN-PMCA interaction happens between the N-terminal region of aSN and the acidic phospholipid binding site of PMCA.

A. Schemes of aSN and its N- and C-terminal truncation variants. Bars represent facultatively helical regions, lines - constitutively disordered regions. 
B. N-terminus is the most important region of aSN for the activation of PMCA. PMCA1d fold activation by titrated full-length aSN and truncated variants. The experiment was performed in presence of $1.8 \mu \mathrm{M}$ free $\mathrm{Ca}^{2+}$ and brain lipid extract was used for the PMCA relipidation. Data are a result of at least three independent activity assays; mean \pm SEM.

C. Sequence comparison of full-length PMCA2w/a and its deletion mutants, missing large fragments of the ATM3 loop: $\Delta 292-383, \Delta 298-383 \Delta 298-372$. The blue background indicates the acidic phospholipid-binding site.

D. The PMCA's acidic phospholipid binding site preceding the transmembrane domain 3 is crucial for the activation by the aSN. Top - The PMCA's specific activity in presence of $1.8 \mu \mathrm{M}$ free $\mathrm{Ca}^{2+}$. PMCA2w/a and mutants were relipidated in the brain lipid extract and the full-length monomeric aSN was used as the activator. Bottom - baseline-corrected data displayed as fold activation of PMCA by aSN. Data are the result of at least three independent measurements; mean \pm SEM.

E. Proposed mechanism of the PMCA-aSN interaction. In presence of acidic membrane phospholipids (red) the N-terminal region of a-SN can strongly interact with both the plasma membrane and the acidic lipid-binding site of PMCA, leading to activation of PMCA.

\section{The activation by aSN is specific to mammalian calcium pump}

PMCA activation by aSN is observed both for the ubiquitous PMCA1 and the more tissuespecific PMCA2 and was also verified for the C-terminally truncated PMCA4x isoform (Supplementary figure 5), which likely colocalizes with aSN in synaptic terminals of retinal neurons (Bodis-Wollner et al., 2014; Cali et al., 2017; Krizaj et al., 2002). The broad impact on PMCA isoforms raised the question of whether the stimulatory effect is at all specific to PMCA.

Interestingly, we found that rabbit SERCAla is also activated by the aSN monomer in the same lipid-dependent manner as PMCA (Figure 5A). However, the effect was statistically relevant only in low $(60 \mathrm{nM})$ free $\mathrm{Ca}^{2+}$ concentration, whereas at high $1.8 \mu \mathrm{M}$ free $\mathrm{Ca}^{2+}$, SERCA was activated to a lower fold. This indicates that aSN may also modulate the apparent $\mathrm{Ca}^{2+}$ affinity of SERCA. With continuous ER extending into presynaptic compartments (Singh et al., 2021; Wu et al., 2017) this is also of relevance to aSN function, and most likely it is also related to the previously reported activation by aSN oligomers (Betzer et al., 2018).

Next, we turned to a plant homolog of PMCA, namely the autoinhibited calcium ATPase of Arabidopsis thaliana (ACA8), belonging to the same P2B subfamily of P-type ATPase (Axelsen and Palmgren, 2001), but not coexisting with aSN, which is only found in vertebrates. Structurally, ACA 8 and PMCA differ in the localization of the autoinhibitory domain, which is situated on the N-terminus of ACA8 and the C-terminus in PMCAs, and ACA8 is lacking the long, disordered loop for the A-TM3 linker with the putative lipid-binding site in PMCA (Brini et al., 2010; Brodin et al., 1992; Pinto Fde and Adamo, 2002). In the presence of brain lipid extract ACA8 is not activated by aSN, but rather inhibited, and the effect is partially reversed by CaM (Figure 5B). In the presence of soy PC, the pump is autoinhibited and can be activated by CaM, but not aSN.

Furthermore, the human Na.K-ATPase ( $\alpha 1$ isoform) was not activated by aSN (Figure 5C). These results suggest the specificity of the interaction for mammalian $\mathrm{Ca}^{2+}$-ATPases. 

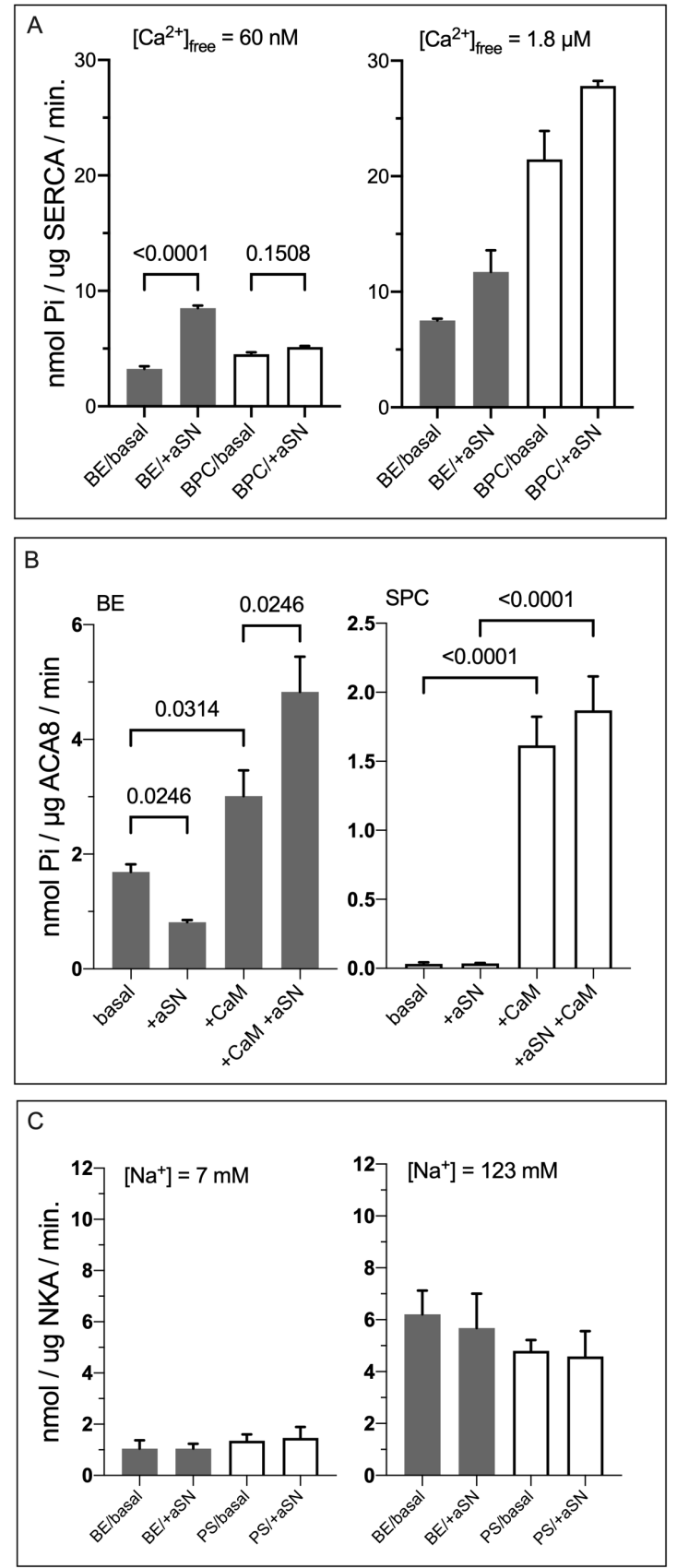

Figure 5. Activation by aSN appears specific to mammalian calcium pumps.

A. In presence of acidic lipids alpha-synuclein activates rabbit SERCA1a in low $\left[\mathrm{Ca}^{2+}\right]_{\text {free }}(60 \mathrm{nM})$. Bars show SERCA activity without or with a-SN when the pump is relipidated in BE (filled bars) or brain BPC (empty bars). $\left[\mathrm{Ca}^{2+}\right]$ free is $60 \mathrm{nM}$ (on the left) and $1.8 \mu \mathrm{M}$ (on the right).

B. Plant calcium ATPase, ACA8 is not activated by aSN. Bars show the activity of ACA8 in presence of 1.8 $\mu \mathrm{M} \mathrm{Ca}^{2+}$ and conditions: without protein partners, with a-SN, with calmodulin $(\mathrm{CaM})$, and with both. PMCA was relipidated in BE (left, filled bars) or soy PC (SPC) (right, empty bars).

C. Human sodium-potassium ATPase (NKA) is not activated by aSN. Bars show the activity of NKA in presence of 7 or $63 \mathrm{nM} \mathrm{Na}^{+}$(right and left graphs, respectively) without or with aSN. NKA was relipidated either in phosphatidylserine (PS) or brain extract (BE).

Everywhere aSN or $\mathrm{CaM}$ are present, the concentrations are $5.6 \mu \mathrm{M}$ or $1.2 \mu \mathrm{M}$, respectively. No-substrate background values were subtracted. Data from at least three independent experiments are expressed as mean \pm SEM. Statistical analysis is conducted as multiple comparisons with one-way ANOVA combined with Sidak post hoc test. 


\section{DISCUSSION}

Here we show a strong, activating effect of soluble, monomeric alpha-synuclein on PMCAs (plasma membrane $\mathrm{Ca}^{2+}$-ATPases) and to a certain degree also SERCA (sarco-/endoplasmic reticulum $\mathrm{Ca}^{2+}$-ATPase). Our results suggest that the physiological role of aSN is stimulation of calcium clearance by increasing $\mathrm{V}_{\max }$ and/or apparent calcium affinity of the active transporters. Due to the very specific localization of aSN, this mechanism would be restricted to specialized cells or cellular compartments like presynaptic termini of neurons. This is supported by our proximity ligation study showing an intracellular interaction of PMCA and aSN in nerve terminals, and by experiments in primary neurons where we show that aSN increases calcium expulsion after depolarization.

The charge of phospholipids in the PMCA environment has a great influence on its regulation, and acidic lipids alone can activate PMCA to some extent via specific interaction sites (Carafoli and Stauffer, 1994; Lopreiato et al., 2014; Strehler, 2015). In a similar way to the recently found inhibition by tau protein (Berrocal et al., 2017), we found the effect of aSN on PMCA is enabled by negatively charged and not neutral phospholipids. The opposite lipid dependence is observed for CaM, consistent with earlier findings (Lopreiato et al., 2014; Niggli et al., 1981a; Niggli et al., 1981b). The composition of the local lipid environment could potentially switch PMCA modes from being responsive either to aSN or CaM.

With its strong effect and accumulation at presynaptic compartments, aSN would allow for a rapid, stimulated response to incoming calcium signals and integration with other calciumsensitive presynaptic functions such as neurotransmitter release. This, together with aSN activating independently of the autoinhibitory C-terminal tail of PMCA, suggests aSN complements $\mathrm{CaM}$ as the activator of PMCA in this specific neuronal compartment.

The alternative splicing of PMCA generates many variants among isoforms and has been for a long time proposed to be a mechanism of fine-tuning of calcium handling in cells affecting expression, localization, and membrane trafficking to specific compartments (Antalffy et al., 2011; Krebs, 2015; Strehler and Zacharias, 2001). Splice site "A" variants of PMCA (w,x,y,z) differ in the size of the lengthy and disordered A-TM3 linker (Fig. 3C). That region was not resolved in a cryo-EM structure of PMCA1 (Gong et al., 2018), and its impact on the enzymatic properties is not clear, but the proximity to the membrane interface and cytosolic $\mathrm{Ca}^{2+}$ entry suggests a modulatory role of the A-TM3 loop; also its involvement in PMCA-lipid interaction and membrane trafficking has been proposed (Adamo and Penniston, 1992; Antalffy et al., 2011; Enyedi and Strehler, 2011). Our mutational studies indeed indicate the modulation of the activity. In PMCA, the deletion of the intrinsically disordered A-TM3 loop (deleting residues 
298-383 in PMCA2w/a) retained basal enzymatic function, but the protein was unresponsive to aSN. However, the stimulation was preserved in a $\Delta 298-372$ construct, where 11 residues upstream of the TM3 are not deleted. This fragment, highly specific in sequence to PMCA, is rich in positive charge and was previously shown to interact with acidic lipids (Adamo and Penniston, 1992; Pinto Fde and Adamo, 2002). Additionally, this construct showed increased basal activity, which may indicate a role of the A-TM3 linker in some level of autoinhibition that complements the autoinhibitory domain at the C-terminal end of PMCA.

The correlations found between aSN expression level and splicing events at the "C" site of PMCA isoforms 1 and 4 suggest that an "aSN/CaM" balance of PMCA activation can be managed by alternative splicing, leading to the synthesis of less CaM-sensitive variants in tissues destined for aSN activity. Variations obtained at the splice site "A" of PMCA2 suggest preference for variant " $w$ " in low-aSN-expressing tissues and shorter variants " $\mathrm{x}$ " and " $\mathrm{z}$ " being expressed together with increasing aSN level. In the activity assay, the PMCA2 splice variants responded differently to aSN: PMCA2w/a was the only one having its apparent calcium affinity increased by aSN, but on the other hand, it was stimulated to the lowest maximum activity and had relatively low apparent calcium affinity in a basal (non-activated) state. The longest ATM3 linker of PMCA2w can be associated with more flexibility in the regulation of its affinity and capacity. In PMCA2"x" and "z" only the capacity was stimulated. The last two variants would be expressed in aSN-abundant tissues with potential demand for higher capacity calcium extrusion.

The specific function of soluble, monomeric aSN is debated (Cheng et al., 2011; Sulzer and Edwards, 2019), but preferential association to membranes with acidic phospholipids is a recurring theme (Davidson et al., 1998; Jo et al., 2000). The ability to activate PMCA appears strongly related to its lipid-binding properties. The aSN A30P mutant form, associated with familial early-onset Parkinson's Disease, is known for reduced ability to bind acidic phospholipids (Jo et al., 2002), and that correlates with the weaker PMCA activation. Similar dependence emerges from the characteristics of the N-terminus of aSN. Monomeric aSN interacts with acidic phospholipids via the $\sim 93$ residues long $\mathrm{N}$-terminal part adopting amphipathic helical segments (Ulmer et al., 2005; Uversky and Eliezer, 2009). The aSN binding to lipid bilayers has been proposed to be maintained by avidity between 1-14 region, strongly interacting and inserting into the lipid bilayer, and the downstream amphipathic helix having weak surface binding capacity (Cholak et al., 2020). Indeed, here we observed a gradual loss of the PMCA activating properties by deletion of 1-14 or 1-29 residues, with the $\Delta(1-29)$ construct being almost incapable of activating PMCA. 
From a structural and mechanistic point of view, we propose that PMCA is activated by interaction between the N-terminus of the monomeric aSN and the acidic lipid binding site of PMCA, located at the C-terminal side of the disordered A-TM3 linker. The bulk part of the loop can play a tuning role together with the disordered C-terminal tail of aSN. With lowaffinity $\mathrm{Ca}^{2+}$-binding properties $\left(\mathrm{K}_{\mathrm{d}} \sim 21 \mu \mathrm{M}\right)$ (Lautenschlager et al., 2018), this region of aSN could potentially increase the local concentration of $\mathrm{Ca}^{2+}$ at the cytosolic entry site of PMCA. It appears to play a role in PMCA activation capacity-wise but does not have a visible impact on apparent calcium affinity.

The PMCA's lipid-binding site is likely functionalized by the many positively charged residues in the 11-residue sequence defining it (RKKASMHKKEK, see also Figure S4). The equivalent sequence in the plant orthologue ACA8 (MASISEDNGEE, residue 352-362) is negatively charged, as is the $\mathrm{N}$-terminal part of the A-TM3 linker in the PMCA2- $\Delta 298-383$ deletion construct (TLLGAGGEEEE). Both show significant basal activity in presence of acidic lipids but are not activated by aSN. The corresponding region in SERCA (RDQMAATEQDK, residue 236 to 246 of rabbit SERCA1a) shows a mixed positive and negative charge, which may explain a mild activation by aSN and negatively charged lipids.

The function of aSN oligomers and fibrils has been thoroughly studied and among a plethora of cytotoxic capacities they have been linked to calcium dysregulation (Betzer et al., 2018; Danzer et al., 2007; Rcom-H'cheo-Gauthier et al., 2016). We show here that native, monomeric aSN exerts a strongly activating effect on PMCA, whereas oligomeric aSN was weaker and perhaps even just ascribed to the small amount of released monomer (Figure S2).

This novel function of aSN is relevant to calcium homeostasis of neurons and specifically presynaptic compartments; however, potentially affects also extracellular environment, where the exchange of each calcium ion for two protons by PMCA leads to formation of transient alkaline nanodomains of the synaptic cleft with consequences to postsynaptic NMDAR fluxes (Chen and Chesler, 2015; Feghhi et al., 2021).

The role of aSN in hematopoietic system has been discussed recently (Pei and Maitta, 2019). The interaction with PMCA can be potentially important to calcium especially in red blood cells, where aSN is very abundant (Barbour et al., 2008). Erythrocytes lack ER and mitochondria, and their calcium homeostasis depends solely on PMCA. The affinity-increasing effect of abundant aSN on PMCA could explain a very low resting calcium level at 30-60 nM observed in red blood cells (Bogdanova et al., 2013).

In the presynapse, PMCA-dependent control of the local calcium-depletion zones could be enforced by aSN and acidic lipids. This may for instance have importance for synaptic 
vesicle release and recycling where PMCA was proposed to functionally separate simultaneous calcium signals (Krick et al., 2021). Oligomerization and aggregation/fibrillation of aSN on the other hand would impair this function and lead to impaired calcium handling. Our finding can then also have implications regarding aSN pathology. Lipid-related dysfunctions have been linked to Parkinson's Disease (Fanning et al., 2020). Early manifestations of PD could result from calcium dyshomeostasis caused by loss of function by aggregating aSN or lipid-affinity affecting mutations like A30P, which leads to early neuronal dysfunctions (Kruger et al., 2001).

\section{ACKNOWLEDGEMENTS}

We thank Torben Heick Jensen and Thomas Gonatopoulos-Pournatzis for advice on transcriptomics data analysis. We are grateful for biosamples and valuable discussion to Michael Habeck for Na,K-ATPase, Thomas Lykke-Møller Sørensen, Anne Lillevang and Claus Olesen for SERCA, and Annette Eva Langkilde, Birthe B. Kragelund and Estella A. Newcombe for $\Delta 14$ and 1-61 alpha-synuclein. We thank Joseph A. Lyons and Magnus Kjærgaard for valuable discussions on membrane proteins and intrinsically disordered proteins, and Robert Edwards for valuable discussion on alpha-synuclein. We are grateful to Anna Marie Nielsen, Tetyana Klymchuk for technical assistance.

This work was supported by a "Mobility plus, $3^{\text {rd }}$ edition" fellowship grant of the Polish Ministry of Science and Higher Education to A.K., a postdoctoral research grant from the Extremadura Province (PO17009) to M.C.B., by a PhD stipend from the Aarhus Graduate School of Science at Aarhus University to S.T.L., by a collaborative grant from H. Lundbeck A/S to P.H.J., by Lundbeck Foundation grants R223-2015-4222 for P.H.J. and R248-20162518 for Danish Research Institute of Translational Neuroscience-DANDRITE, Nordic-EMBL Partnership for Molecular to P.N and P.H.J., and by funds from a project 2 research grant from the Independent Research Fund Denmark and the Brainstruc research center and a professorship grant of the Lundbeck Foundation to P.N.

\section{AUTHOR CONTRIBUTIONS}

P.H.J. and P.N conceived PMCA studies and P.H.J. and A.K. the aSN interaction studies; A.K designed constructs and experiments, except co-immunoprecipitation, proximity ligation assay, and calcium measurements, which were designed, performed and analyzed by C.B. and E.G.; A.K. developed PMCA expression and purification protocols, assisted also by S.T.L. and M.C.B.; C.B. performed the expression and purification of aSN; A.K., S.T.L., and M.C.B. performed and analyzed ATPase activity assays; S.T.L. expressed and purified ACA8 and 
calmodulin, and performed the analysis of the transcriptomics data; A.K. wrote the manuscript with P.N. and with inputs from all authors.

\section{DECLARATION OF INTEREST}

The authors declare no competing interests. 


\section{METHODS}

\section{RESOURCE AVAILABILITY}

\section{Lead contact}

Requests for further information, resources, and reagents should be directed to and will be fulfilled by the lead contact, Poul Nissen (pn@mbg.au.dk)

\section{Materials availability}

All plasmids generated in this study are available from the Lead Contact without restriction. Reagents used in the study were of general use and from commercial sources.

\section{Data and code availability}

The transcriptomics data are publicly available at https://vastdb.crg.eu/ All original code written in $\mathrm{R}$ for the analysis is available in this paper's supplemental information. The remaining data reported in this paper will be shared by the lead contact upon request.

\section{METHOD DETAILS}

\section{Co-immunoprecipitation assay}

Brains from C57BL/6 mice (Janvier Labs) or aSN-KO (Sncatm1Rosl (C57BL/6), The Jackson Laboratory) were homogenized in 7x w/v homogenization buffer ( $320 \mathrm{mM}$ sucrose, 4 mM HEPES-NaOH, 2 mM EDTA, and Complete protease inhibitor mix (Roche), pH 7.4) using a loose-fitting glass-Teflon homogenizer (10 up-and-down strokes, $700 \mathrm{rpm}$ ). Debris was removed from the homogenate by centrifugation for $10 \mathrm{~min}$ at $1000 \mathrm{x} \mathrm{g}$ in a Sorvall $\mathrm{RC} 5 \mathrm{C}$ plus centrifuge. The resulting supernatant was centrifuged for $1 \mathrm{~h}$ at $100000 \mathrm{x}$. The supernatant was removed, and the pellet was resuspended in the original volume (7x) of RIPA (50 mM Tris pH 7.4, $159 \mathrm{mM} \mathrm{NaCl}, 1 \%$ Triton X-100, 2 mM EDTA, 0.5\% sodium deoxycholate, $0.1 \%$ SDS) for $3 \mathrm{~h}$, whereafter samples were spun at $20000 \mathrm{xg}$, for $30 \mathrm{~min}$ at $4{ }^{\circ} \mathrm{C}$. Protein concentration was measured using the bicinchoninic acid assay. aSN oligomer or monomer $(2 \mu \mathrm{g} / \mathrm{ml})$ was mixed with $0.5 \mathrm{mg} / \mathrm{ml}$ aSN-KO mouse brain homogenate diluted in PBS, $0.5 \%$ Triton X-100 and incubated overnight at $4^{\circ} \mathrm{C}$. aSN binding (ASY-1) or control (non-immune $\operatorname{IgG}$ ) was performed as previously described (Betzer et al., 2015). The samples were incubated for $2 \mathrm{~h}$ with rotation. The Sepharose beads were isolated and washed twice with PBS, $0.5 \%$ Triton X100 , and Co-IP proteins were eluted by incubation in a non-reducing SDS loading buffer at room temperature. Proteins were resolved on 10-16\% gradient SDS-PAGE under reducing conditions followed by immunoblotting for PMCA (primary antibody: 5F10 anti-pan-PMCA, Abcam, ab2825, secondary antibody: anti-mouse-HRP, Dako) and aSN (primary antibody: 
anti-Syn-1, BD Transduction Laboratory, secondary antibody: anti-mouse-HRP, Dako). The interaction between endogenous aSN and the endogenous PMCA was studied in the extracts from $\mathrm{C} 57 \mathrm{BL} / 6$ mice as described above.

\section{Primary hippocampal neuronal cultures and cytosolic $\mathrm{Ca}^{2+}$ measurements}

Primary hippocampal neurons were cultured from newborn (P0) aSN-KO mice (Sncatm1Rosl (C57BL/6), The Jackson Laboratory). Hippocampi were dissected in ice-cold Hank's balanced salt solution, dissociated in $20 \mathrm{U} / \mathrm{ml}$ papain in Hibernate A medium (Gibco) supplemented with $1 \times B 27$ and $0.3 \mathrm{~g} / \mathrm{L} \mathrm{l-glutamine}$ for $20 \mathrm{~min}$ at $37^{\circ} \mathrm{C}$, washed twice, and triturated in plating medium [MEM (Gibco) supplemented with $5 \mathrm{~g} / 1$ glucose, $0.2 \mathrm{~g} / 1 \mathrm{NaHCO}$, $0.1 \mathrm{~g} / 1$ transferrin, $0.25 \mathrm{~g} / 1$ insulin, $0.3 \mathrm{~g} / \mathrm{L}$ l-glutamine, and $10 \%$ fetal bovine calf serum (heatinactivated)]. Hippocampal neurons were seeded on Matrigel ${ }^{\circledR}$ matrix (Corning $\left.{ }^{\circledR}\right)$-coated coverslips. After $24 \mathrm{~h}$, the medium was changed to growth medium [MEM supplemented with $5 \mathrm{~g} / 1$ glucose, $0.2 \mathrm{~g} / 1 \mathrm{NaHCO}_{3}, 0.1 \mathrm{~g} / 1$ Transferrin, $0.075 \mathrm{~g} / \mathrm{L}$ 1-glutamine, $1 \times \mathrm{B}-27$ supplement, $2 \mu \mathrm{M}$ cytosine arabinoside and 5\% fetal bovine calf serum (heat-inactivated)].

At culture day 6 (DIV 6), the neurons were transfected by lipofectamine 3000 with Bicistronic vectors coding for mCherry and aSN or mCherry under synapsin promotor according to manufacturer's instructions. At culture day 8 cytosolic $\mathrm{Ca}^{2+}$ levels in primary neurons were determined using the $\mathrm{Ca}^{2+}$-sensitive fluorescent indicator Fura-2-AM (Molecular Probes/Invitrogen). Cells were loaded with Fura-2 in sterile-filtered HEPES-buffered saline (HBS: 20 mM HEPES, $150 \mathrm{mM} \mathrm{NaCl}, 5 \mathrm{mM} \mathrm{KCl}, 1 \mathrm{mM} \mathrm{CaCl}, 1 \mathrm{mM} \mathrm{MgCl} 2,10 \mathrm{mM}$ glucose, $\mathrm{pH} 7.4$ ) containing $2.5 \mu \mathrm{M}$ Fura-2 AM, $0.04 \%$ pluronic acid, $\mathrm{F} 127$ for $30 \mathrm{~min}$ at $37^{\circ} \mathrm{C}, 5 \% \mathrm{CO}_{2}$. The Fura-2-containing medium was replaced with fresh HBS without Fura-2 and incubated additionally for $30 \mathrm{~min}$. After dye loading and prior microscopic analysis, the culture was moved into a recording buffer with thapsigargin for $5 \mathrm{~min}$., and an area containing 1-3 transfected neurons was found. The fluorescence was measured on an Olympus Scan ${ }^{\wedge} \mathrm{R}$ highcontent microscope using excitation wavelengths at 340 and $380 \mathrm{~nm}$ and emission at $510 \mathrm{~nm}$. The cytosolic $\mathrm{Ca}^{2+}$ levels in single cells were measured by placing a region of interest (ROI) outside the nucleus. The recording was started and at the recording time of 2 min $\mathrm{KCl}$ was added to depolarize the neurons. The calcium response was followed over time until $12 \mathrm{~min}$. The response upon $\mathrm{K}^{+}$induced depolarization was quantified as the Area Under Curve (AUC) from each measured neuron from the 2-12 min. measurement interval. 


\section{Proximity ligation assay (PLA)}

Primary hippocampal neurons were cultured from newborn (P0) C57BL/6 mice (Janvier Labs) as described for aSN-KO. At culture day 14 (DIV14) the neurons were fixed in 4\% paraformaldehyde for $30 \mathrm{~min}$ at room temperature (RT) followed by a wash in PBS and $10 \mathrm{~min}$ permeabilization in $0.1 \%$ Triton $\mathrm{X}-100,50 \mathrm{mM}$ glycine, $3 \mathrm{mM} \mathrm{CaCl}_{2}, 2 \mathrm{mM} \mathrm{MgCl} 2, \mathrm{pH}$ 7.4. Unspecific binding was blocked by 3\% bovine serum albumin in PBS for $1 \mathrm{~h}$ followed by incubation with the primary antibody for $1.5 \mathrm{~h}$ [anti-AS $(1 \mu \mathrm{g} / \mathrm{ml}$, ASY-1(Kragh et al., 2009; Lindersson et al., 2004)), and anti-PMCA 5F10 (1 $\mu \mathrm{g} / \mathrm{ml}$, Abcam, ab2825). The Duolink procedure was conducted according to the manufacturer's instructions with the Duolink ${ }^{\circledR}$ In Situ Red Starter Kit Mouse/Rabbit (Duolink ${ }^{\circledR}$, Sigma-Aldrich), with secondary antibodies contained in the kit. After the PLA staining, the neurons were labeled with synaptophysin (primary antibody - guinea pig anti-synaptophysin 1, (Synaptic Systems \#101004), secondary: goat anti-guinea pig, Alexa Fluor ${ }^{\circledR} 488 \mathrm{~nm}$ (Abcam, ab150185) to visualize the synapses where synuclein normally is located and DAPI for nuclei. Images were obtained using a Zeiss Observer Z1 inverted microscope equipped with ApoTome.2.

\section{Expression and purification of $\alpha$-synuclein}

Recombinant human aSN wild type and mutants were produced in E. coli, and purified as previously described (Huang et al., 2005). Monomeric and oligomeric forms of aSN were produced and isolated as previously described (Betzer et al., 2015). Concentration of all aSN preparations was confirmed by $\mathrm{BCA}$ assay. The proteins were stored in $-80^{\circ} \mathrm{C}$ in a buffer containing $20 \mathrm{mM}$ Tris/HCl, $\mathrm{pH}$ 7.5, $150 \mathrm{mM} \mathrm{KCl}$.

\section{PMCA expression constructs and site-directed mutagenesis}

Codon-optimized genes encoding for human PMCA1d and PMCA2w/a (Genscript) were cloned into pEMBL-yex4 expression plasmids by homologous recombination in S. cerevisiae as described (Drew et al., 2008).

PMCA1d and PMCA2w/a constructs were subjected to site-directed mutagenesis using QuikChange site-directed mutagenesis kit according to the manufacturer's protocol (Agilent). To obtain an inactive mutant D475N (the autophosphorylation site) mutagenetic primers were designed using PrimerX online tool (https://www.bioinformatics.org/primerx). To obtain Cterminally truncated PMCA1, an internal TEV cleavage site was introduced to the original construct. Primers for the insertion of codon-optimized TEV site sequence and the large 
deletions in PMCA2 were designed according to described methods (Liu and Naismith, 2008). The integrity of all constructs was verified by DNA sequencing (Eurofins).

\section{Expression of recombinant PMCAs in S. cerevisiae}

All circular vectors were introduced into yeast cells using PEG/LiAc/ssDNA-mediated transformation (Gietz and Schiestl, 2007). All recombinant PMCAs were expressed in $S$. cerevisiae strain K616, depleted of the native calcium ATPases (MAT $\alpha$ pmr1::HIS3 pmc1::TRP1 cnb::LEU2 ura3-1) (Cunningham and Fink, 1994). All culture media were supplemented with $10 \mathrm{mM} \mathrm{CaCl}_{2}$, as it is a necessary survival condition for the strain to maintain internal stores. For expression culture, $100 \mathrm{ml}$ of uracil-deficient $2 \%$ glucose SD medium was inoculated with a colony of transformed cells and allowed to grow for $24 \mathrm{~h}$ in $30^{\circ} \mathrm{C}$ in a $120 \mathrm{rpm}$ shaking incubator. The preculture was used for inoculation of 9 liters of Ura SD medium with $1 \%$ glucose and $40 \mathrm{mg} / \mathrm{L}$ adenine hemisulfate, starting from $\mathrm{OD}_{450}$ of 0.05 . After 22 hours, when all glucose in the medium was used and the culture reached $\mathrm{OD}_{450}$ of $\sim 5$, the expression was induced by $2 \%$ galactose, and the culture was supplemented with YP medium and $40 \mathrm{mg} / \mathrm{L}$ adenine hemisulfate. Cells were harvested after 18-20 hours by centrifugation, washed with water and TEKS buffer $(50 \mathrm{mM}$ Tris/ $\mathrm{HCl} \mathrm{pH}$ 7.6, $2 \mathrm{mM}$ EDTA, $100 \mathrm{mM} \mathrm{KCl}, 0.6 \mathrm{M}$ D-sorbitol), and resuspended in TESin buffer (50 mM Tris/ $\mathrm{HCl}, 2 \mathrm{mM}$ EDTA, 0.6 M D-sorbitol with the addition of PMSF and protease inhibitor. The typical yield was $\sim 15 \mathrm{~g}$ of cells per liter of culture.

\section{Purification of PMCAs}

The cells were disrupted in a pulverisette grinder with an equal volume of $0.5 \mathrm{~mm}$ glass beads $\left(4^{\circ} \mathrm{C}, 450 \mathrm{rpm}, 4\right.$ cycles of $3 \mathrm{~min}$. millings, and $1 \mathrm{~min}$. pause). The material was centrifuged for $20 \mathrm{~min}$ at $2000 \mathrm{x}$ g to remove beads and uncracked cells. The supernatant (S1) was centrifuged for $30 \mathrm{~min}$ at $20000 \mathrm{x}$ g for further removal of cell debris. The supernatant (S2) was subjected to ultracentrifugation for $3 \mathrm{~h}$ at $150000 \mathrm{x}$ g. Pelleted membranes were

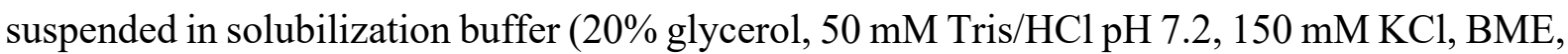
PMSF, protease inhibitors) and homogenized in a Dounce glass homogenizer. Membranes were flash-frozen in liquid nitrogen and stored at $-80^{\circ} \mathrm{C}$ until further processing.

Membranes were diluted with solubilization buffer to $5 \mathrm{mg} / \mathrm{ml}$ total protein concentration (measured with Bradford assay) and solubilized in 1\% DDM and $0.2 \%$ cholesteryl hemisuccinate for $1 \mathrm{~h}$ by stirring in $4^{\circ} \mathrm{C}$, followed by ultracentrifugation at $150000 \mathrm{x}$ g for $1 \mathrm{~h}$ 
to pellet unsolubilized material. The supernatant was supplemented with $4 \mathrm{mM} \mathrm{CaCl}_{2}$ and stirred for $2 \mathrm{~h}$ in $4^{\circ} \mathrm{C}$ with calmodulin sepharose beads (GE Healthcare), pre-equilibrated with binding buffer $(50 \mathrm{mM}$ Tris $/ \mathrm{HCl} \mathrm{pH} 7.2,150 \mathrm{mM} \mathrm{KCl}, 0.017 \%$ w/v DDM, 10\% glycerol, 2 $\mathrm{mM} \mathrm{CaCl}_{2}$ ). The beads were packed onto a gravity flow column and washed with 10-15 column volumes of binding buffer. The protein was eluted a buffer containing $50 \mathrm{mM}$ Tris/ $\mathrm{HCl} \mathrm{pH} \mathrm{7.2,}$ $150 \mathrm{mM} \mathrm{KCl}, 0.017 \% \mathrm{w} / \mathrm{v}$ DDM, 10\% glycerol, 2 mM EGTA. EGTA stock solution (200 mM) was beforehand $\mathrm{pH}$-adjusted with $\mathrm{KOH}$ to $\sim 7.5$. The fractions containing the protein of interest were pooled and concentrated to $\sim 3 \mathrm{mg} / \mathrm{mL}$ using a $100 \mathrm{kDa}$ MWCO Vivaspin protein concentrator (GE Healthcare). The concentrated protein was subjected to size exclusion chromatography on Superose 6 increase 10/300 column (GE Healthcare).

To produce C-terminally truncated PMCA1, the PMCA1d construct with a tobacco etch virus (TEV) protease cleavage site inserted next to $\mathrm{H} 1074$ just before the autoinhibitory domain was used. The protein was cleaved with TEV protease while bound to the CaM sepharose beads. After overnight incubation with the protease at $4^{\circ} \mathrm{C}$, the C-terminally truncated PMCA was collected in the flow-through fraction and run over a $\mathrm{Ni}^{2+}$ affinity column to bind the His-tagged protease. The flow-through was concentrated to $\sim 3 \mathrm{mg} / \mathrm{mL}$ (100kDa MWCO) and subjected to size exclusion chromatography on Superdex 200 increase 10/300 column (GE healthcare) in size exclusion buffer containing $50 \mathrm{mM}$ Tris/ $\mathrm{HCl} \mathrm{pH}$ 7.2, $150 \mathrm{mM} \mathrm{KCl,} \mathrm{0.017 \%} \mathrm{w/v} \mathrm{DDM,}$ $10 \%$ glycerol. Peak fractions of PMCAs were pooled, concentrated to $1-1,5 \mathrm{mg} / \mathrm{ml}$ if necessary, using a $100 \mathrm{kDa}$ MWCO Vivaspin protein concentrator (Sartorius), aliquoted, and flash-frozen in liquid $\mathrm{N}_{2}$ and stored at $-80^{\circ} \mathrm{C}$ for further procedures. The purity of the proteins was analyzed by SDS-PAGE (Supplementary Fig. 1).

\section{Expression and purification of calmodulin}

Mammalian calmodulin $(\mathrm{CaM})$ in the $\mathrm{pET} 15 \mathrm{~b}$ vector was transformed into E. coli strain $\mathrm{C} 41$ and grown in $\mathrm{LB}$ media at $37^{\circ} \mathrm{C}$. Induction was performed with IPTG at an $\mathrm{OD}_{600}$ of 0.6. After 16 hours of growth at $21^{\circ} \mathrm{C}$ cells were harvested by centrifugation at $3000 \mathrm{x} \mathrm{g}$, resuspended in lysis buffer $(20 \mathrm{mM}$ Tris- $\mathrm{HCl} \mathrm{pH} 7.5,1 \mathrm{mM}$ EDTA, complete protease inhibitor), and lysed using sonication. The lysate was centrifuged for an hour at $20000 \mathrm{xg}$. The supernatant was mixed 1:1 with wash buffer $(20 \mathrm{mM}$ Tris- $\mathrm{HCl} \mathrm{pH} 7.5,10 \mathrm{mM} \mathrm{CaCl} 2)$ and loaded onto a pre-equilibrated (wash buffer) gravity flow phenyl sepharose column. The column was washed with $5 \mathrm{CV}$ wash buffer, $5 \mathrm{CV}$ high salt wash buffer $(20 \mathrm{mM}$ Tris-HCl pH 7.5, $500 \mathrm{mM} \mathrm{NaCl}, 10 \mathrm{mM} \mathrm{CaCl}$ ), and $5 \mathrm{CV}$ wash buffer. CaM was eluted in elution buffer (50 mM Tris- $\mathrm{HCl} \mathrm{pH} 7.5,150 \mathrm{mM} \mathrm{NaCl}, 10 \mathrm{mM}$ EGTA). Before use in activity assays CaM 
buffer was exchanged to $20 \mathrm{mM}$ Tris- $\mathrm{HCl} \mathrm{pH} 7.5,150 \mathrm{mM} \mathrm{KCl}$ using PD MiniTrap G25 desalting column (GE Healthcare), aliquoted and stored in $-80^{\circ} \mathrm{C}$.

\section{Expression and purification of ACA8}

ACA8 expression and membrane isolation was performed similarly to the PMCA isoforms. ACA8 was solubilized in a DDM solubilisation buffer $(50 \mathrm{mM}$ Tris- $\mathrm{HCl} \mathrm{pH} 7.6,100 \mathrm{mM} \mathrm{KCl}$, $20 \%$ glycerol, $3 \mathrm{mM} \mathrm{MgCl} 2,5 \mathrm{mM} \beta$-mercaptoethanol ( $\beta \mathrm{ME}), 7.5 \mathrm{mg} / \mathrm{mL}$ DDM, $1 \mathrm{mM}$ PMSF and $1 \mu \mathrm{g} / \mathrm{mL}$ of chymostatin, pepstatin $\mathrm{A}$ and leupeptin). Purification was achieved through $\mathrm{Ni}^{2+}$ affinity followed by size exclusion chromatography. ACA 8 was bound to a $\mathrm{Ni}^{2+}$-affinity column in low imidazole buffer $(50 \mathrm{mM}$ Tris- $\mathrm{HCl}$ pH 7.5, $10 \mathrm{mM}$ imidazole, $200 \mathrm{mM} \mathrm{KCl}, 10$ $\%$ glycerol, $3 \mathrm{mM} \mathrm{MgCl} 2,5 \mathrm{mM} \beta \mathrm{ME}, 0.2 \mathrm{mM} \mathrm{LMNG).} \mathrm{On} \mathrm{the} \mathrm{column} \mathrm{the} \mathrm{ACA} 8$ His-tag was cleaved off with thrombin and ACA8 was eluted in low imidazole buffer. ACA8 was subjected to size exclusion chromatography on a Superose 6 column in a SEC buffer ( $50 \mathrm{mM}$ Tris- $\mathrm{HCl}$ pH 7.5, $200 \mathrm{mM} \mathrm{KCl,} 3 \mathrm{mM} \mathrm{MgCl} 2,10 \%$ glycerol, $0.02 \mathrm{mM}$ LMNG, $5 \mathrm{mM} \beta \mathrm{ME})$. The protein was stored in $-80^{\circ} \mathrm{C}$ in SEC buffer for further experiments.

\section{SERCA and Na, K-ATPase preparations}

SERCA1a from rabbit skeletal muscle was kindly donated by Thomas Lykke-Møller Sørensen, Anne Lillevang and Claus Olesen. The protein preparation was performed according to previously established procedures (Andersen et al., 1985; Sorensen et al., 2004). Na, KATPase was kindly donated by Michael Habeck and Natalya Fedosova. The protein was expressed and purified as described earlier (Habeck et al., 2017)

\section{ATPase activity assay}

The ATPase activity was tested by monitoring the release of free inorganic phosphate from ATP in a molybdenum blue assay (Baginski et al., 1967).

Purified PMCA requires relipidation to regain activity, therefore the samples were preincubated with phospholipids before activity assay. Lipid stock solutions were prepared from powdered lipids as follows: brain lipid extract (from bovine brain Type I, Folch Fraction I, Sigma-Aldrich), brain PC (Avanti), or soy PC (Avanti) were dissolved to a concentration of $20 \mathrm{mg} / \mathrm{ml}$ in a buffer containing $50 \mathrm{mM}$ Tris $\mathrm{pH}$ 7.5, $150 \mathrm{mM} \mathrm{KCl}$ and $1.5 \%$ (w/v) DDM. After cycles of heating $\left(45-50^{\circ} \mathrm{C}\right)$, sonicating, and vortexing, a translucent gel was obtained. Stocks were aliquoted and stored in $-20^{\circ} \mathrm{C}$. Shortly before the assay lipid solution was added to PMCA sample in PMCA:lipid $\mathrm{w} / \mathrm{w}$ ratio 1:5 and mixed by gentle pipetting. After $15 \mathrm{~min}$, the 
relipidated protein was added to a final concentration of $2.5 \mu \mathrm{g} / \mathrm{ml}$ to a reaction buffer containing $50 \mathrm{mM} \mathrm{KNO}_{3}, 5 \mathrm{mM} \mathrm{NaN}_{3}, 0.25 \mathrm{mM} \mathrm{NaMob}, 40 \mathrm{mM}$ BisTris/HEPES pH 7.2, 3 $\mathrm{mM} \mathrm{MgSO} 4,1 \mathrm{mM}$ EGTA and $\mathrm{CaCl}_{2}$ in concentration needed for obtaining the desired concentration of free calcium ions. The free calcium concentration was calculated using Maxchelator - Ca-Mg-ATP-EGTA Calculator. Alpha-synuclein and/or calmodulin were added to the reaction mixture in desired concentrations and corresponding protein storage buffer was added to control samples in equal volume. The reactions were started by adding $3 \mathrm{mM} \mathrm{ATP \text {, }}$ carried out at $37^{\circ} \mathrm{C}$, and stopped after 7 or 11 minutes by adding a stop solution. Stop solution was prepared shortly before every assay by mixing ice-cold solutions A (17 $\mu \mathrm{M}$ ascorbic acid, $0.1 \%$ SDS in $0.5 \mathrm{M} \mathrm{HCl})$ and $\mathrm{B}(0.7 \mu \mathrm{M}$ ammonium molybdate VI tetrahydrate). The absorbance was measured with Victor 3 96-well plate reader (Perkin-Elmer) at $860 \mathrm{~nm}$.

\section{Transcriptomics data analysis}

Data on gene expression for PMCA1-4 and aSN were used. Data on tissue expression and exon incorporation from VastDB for the genes ATP2B1, ATP2B2, ATP2B3, ATP2B4, and SNCA were imported in R. For the PMCA exons of relevance, the "percent spliced-in" (PSI) was correlated to aSN tissue expression. For tissues in specified aSN expression intervals (ranging from 0-190 interval size of 10) the average incorporation of the exons was determined, and the mean PSI was plotted as a function of the aSN expression interval. The code written in $\mathrm{R}$ for the analysis is included in the supplemental information.

\section{Protein disorder prediction}

Protein disorder prediction was performed using NetSurfP 2.0 software developed by the Technical University of Denmark and available at https://services.healthtech.dtu.dk/service.php?NetSurfP-2.0.

\section{Statistical analysis}

Statistics were performed using GraphPad Prism and $\mathrm{P}$ values $<0.05$ were considered significant. The statistical method was described in the figure legends. 


\section{SUPPLEMENTAL INFORMATION TITLES AND LEGENDS}

I
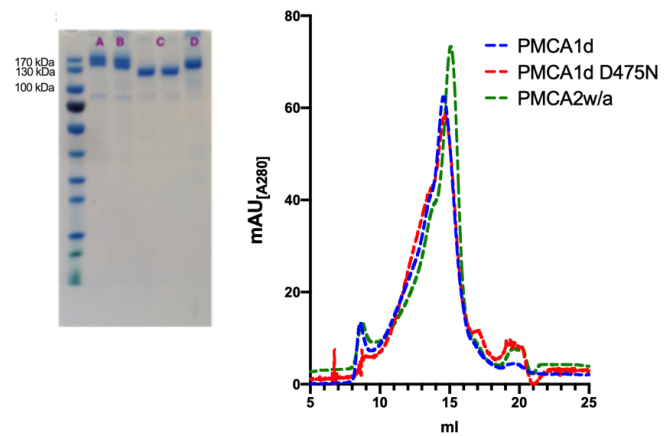

III

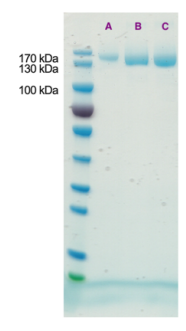

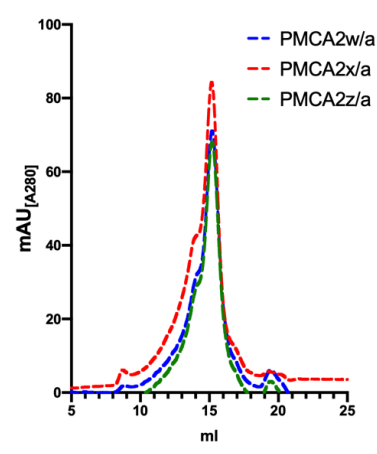


IV

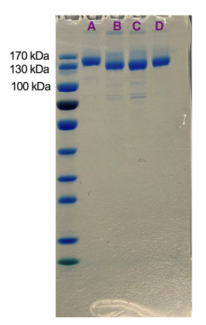

Figure S1. Purification of recombinant PMCAs

I - Left - SDS PAGE of purified PMCAs. (A) PMCA1d, PMCA1d D475N (inactive mutant), PMCA1- $\Delta$ C, (D) PMCA2w/a. Right - Corresponding profiles of the size exclusion chromatography performed on the Superose 6 increase column.

II - Left - SDS-PAGE analysis of the purification of the PMCA1- $\Delta$ C. Full PMCA1d with internal TEV cleavage site at position 1074 was bound to calmodulin sepharose and washed (lane A - a sample of washed beads). After overnight incubation with His-tagged TEV flow-through 1 (FT1, lane B) was collected and beads were washed with buffer in a gravity-flow column (lane C - wash). FT1 was put through a Ni-Sepharose in a gravity-flow column to bind TEV and uncleaved PMCA1 (D - sample of washed sepharose beads). The flow-through from the nickel column was collected, concentrated to $500 \mu \mathrm{L}$, and subjected to size exclusion chromatography on Superdex 200 column. Lane E - peak fraction from the size-exclusion chromatography. Lane F - TEV protease. Right Profile of the size exclusion chromatography of PMCA1- $\Delta$ C performed on the Superdex 200 increase column.

III - Left - SDS PAGE of purified PMCA2 splice variants (A-C) w/a, x/a, z/a. Right - Corresponding profiles of the size exclusion chromatography performed on the Superose 6 increase column.

IV - Left - SDS PAGE of purified PMCA2w/a (A) and its deletion mutants $\Delta 292-383$ (B), $\Delta 298-383$ (C), and $\Delta 298-372$ (D). Right - Corresponding profiles of the size exclusion chromatography performed on the Superose 6 increase column. 


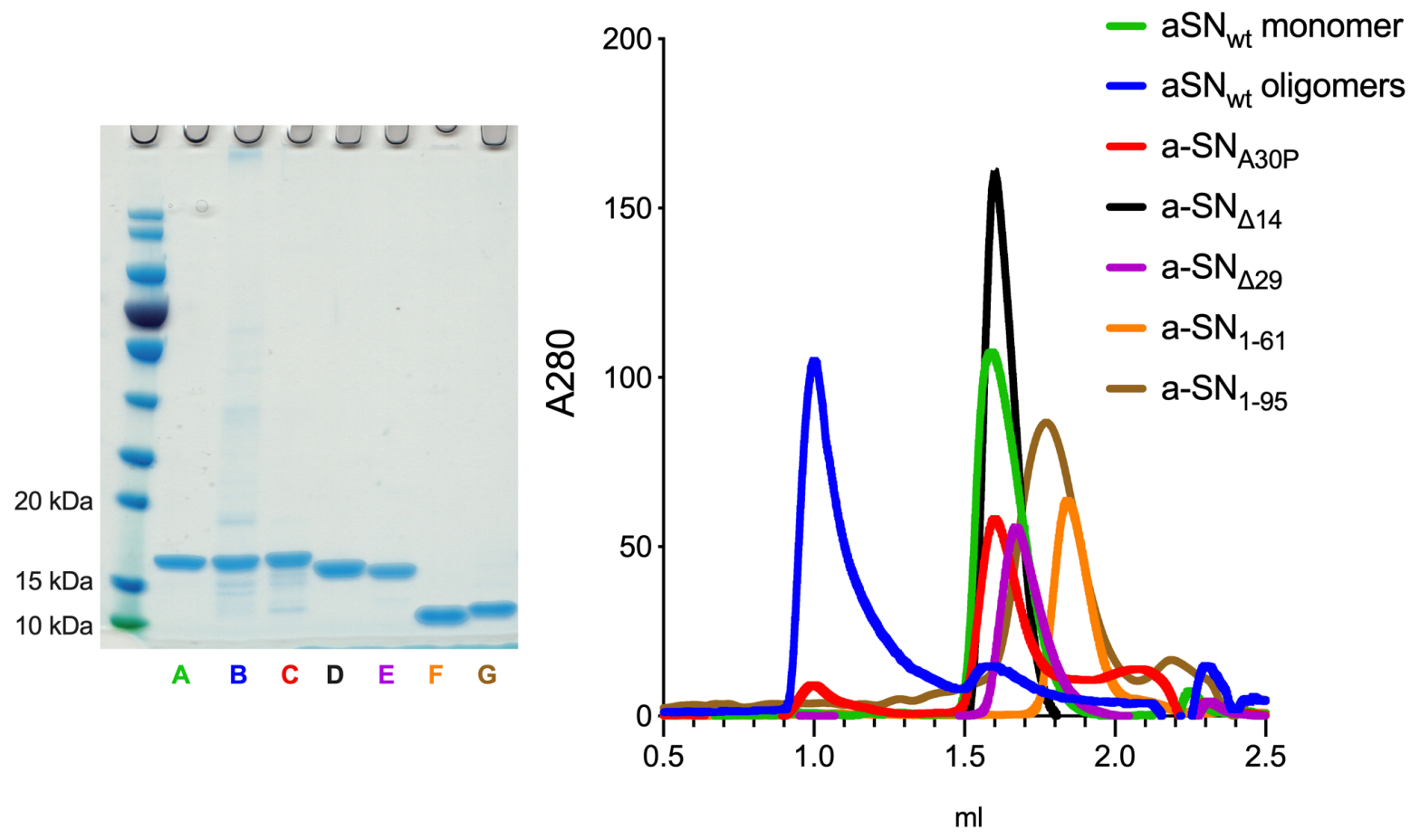

Figure S2. Purity of alpha-synuclein preparations.

On the left - SDS-PAGE analysis A - monomer; B - oligomers; C - A30P; D - $\Delta 14$; E - $\Delta 29$; F - (1-61); G - (195); On the right - size exclusion chromatography analysis performed on Superdex 200 increase 3.2/300 column. Monomeric forms were eluted at volume $>1.5 \mathrm{ml}$ 
A

๑ basal • $\cdot$ +aSN $\cdot \mathbf{a} \cdot$ +CaM

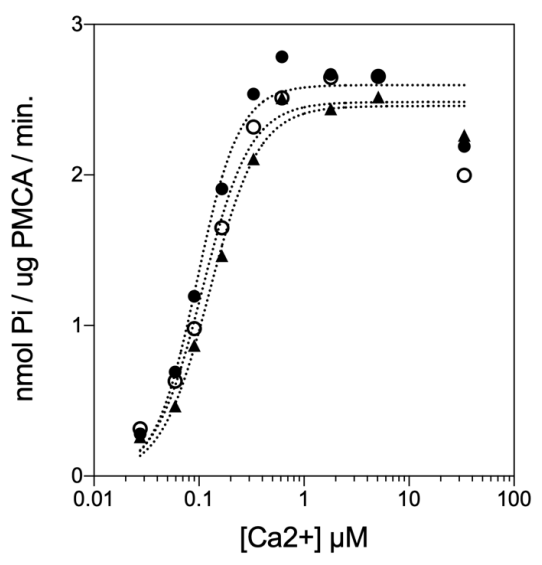

C



B

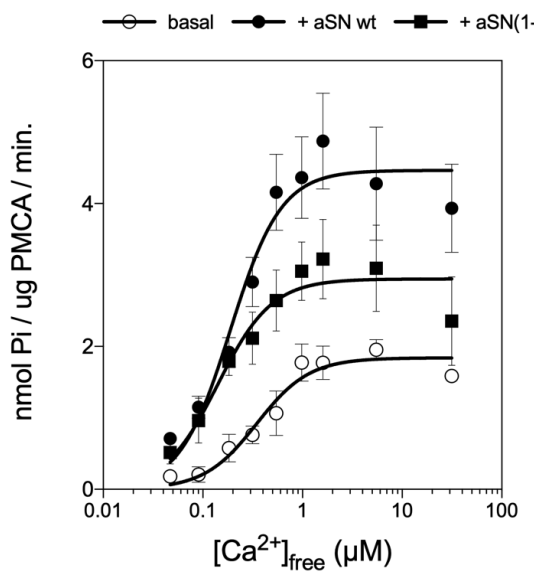

D

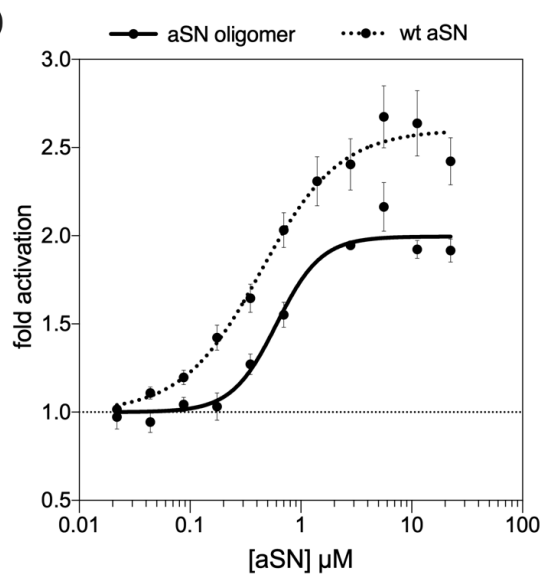

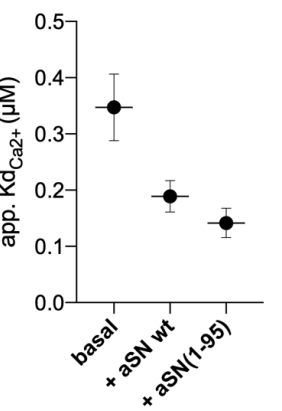

Figure S3.

A - The activity of PMCA1- $\Delta C$ in the presence of brain PC lipids. $\mathrm{Ca}^{2+}$ titrations were performed in absence of an activating partner, in the presence of $2.8 \mu \mathrm{M}$ alpha-synuclein or $1.2 \mu \mathrm{M}$ calmodulin.

$B$ - The effect of full-length and C-terminally truncated $\operatorname{aSN}_{(1-95)}$ on the calcium-dependent activity of PMCA1d. On the left - the calcium titration experiment was performed simultaneously in absence of activating partner (empty circles), in presence of the full-length monomeric a-SN (filled circles) and C-terminally truncated $\operatorname{aSN}_{(1-95)}$ (filled squares). The pump was relipidated in brain lipid extract (BE). On the right - apparent $\mathrm{Kd}_{\mathrm{Ca} 2+}$ values calculated from the fitted Hill plots. The lines are the best fit given by the Hill equation with the apparent $\mathrm{Kd}$ values $(\mu \mathrm{M})$ for $\mathrm{Ca}^{2+}$ as follows: PMCA1d basal (without aSN) $-0.347 \pm 0.059$, with aSNwt $-0.189 \pm 0.028$, with $\operatorname{aSN}_{(1-95)}-0.142 \pm 0.026$

C - The activity of C-terminally truncated PMCA4x (lacking the autoinhibitory domain) was measured as a function of monomeric a-SN concentration in presence of $1.8 \mu \mathrm{M}$ free $\mathrm{Ca}^{2+}$. PMCA was relipidated with brain extract (BE).

D - The effect of oligomeric aSN on the PMCA activity. PMCA1d fold activation by titrated full-length monomeric and oligomeric aSN. Monomeric aSN data, presented here for comparison, is the same as in Figure 4. The assay was performed in presence of $1.8 \mu \mathrm{M}$ free $\mathrm{Ca}^{2+}$ and brain lipid extract was used for the PMCA relipidation. Data are a result of at least three independent activity assays; mean $\pm \mathrm{SEM}$. 
PMCA2 298-372 273 - VTAVGVNSQTGIIFTLLGAGGEEEERKKASMHKKEKSVLQGKLTKLAVQIGKAGLVMSAI-TV

PMCA2 298-383 273 - VTAVGVNSQTGIIFTLLGAGGEEEE--_-.-_-SVLQGKLTKLAVQIGKAGLVMSAI-TV

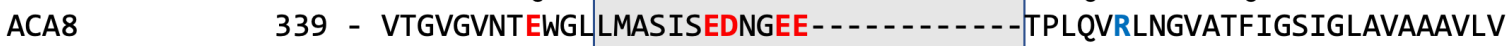

SERCA1a 223 - VATTGVSTEIGKIRDQMAAT-...-.-EQDKTPLQQKLDEFGEQLSKVISLICVAVWL

Supplementary figure 4. Sequence alignment of the A-TM3 linker region and the TM3 domain of PMCA2w/aA298-372, PMCA2w/aA298-383, ACA8 and SERCA1a. Positively charged residues marked blue, negatively - red. Italics show the acidic lipid binding site of PMCA2. Alignment of full sequences was performed with uniport.org, sequences from which presented fragments were taken have the following entry numbers in Uniprot: Q01814-2 (PMCA2w/a, modified for the alignment, to show both PMCA deletion mutants), Q9LF79 (ACA8), and P04191 (SERCA1a).

\section{REFERENCES}

Adamo, H.P., and Penniston, J.T. (1992). New Ca2+ pump isoforms generated by alternative splicing of rPMCA2 mRNA. Biochem J 283 ( Pt 2), 355-359. 10.1042/bj2830355.

Andersen, J.P., Lassen, K., and Moller, J.V. (1985). Changes in Ca2+ affinity related to conformational transitions in the phosphorylated state of soluble monomeric Ca2+-ATPase from sarcoplasmic reticulum. J Biol Chem 260, 371-380.

Antalffy, G., Caride, A.J., Paszty, K., Hegedus, L., Padanyi, R., Strehler, E.E., and Enyedi, A. (2011). Apical localization of PMCA2w/b is enhanced in terminally polarized MDCK cells. Biochem Biophys Res Commun 410, 322-327. 10.1016/j.bbrc.2011.05.147.

Augustine, G.J., Santamaria, F., and Tanaka, K. (2003). Local calcium signaling in neurons. Neuron 40, 331-346. 10.1016/s0896-6273(03)00639-1.

Axelsen, K.B., and Palmgren, M.G. (2001). Inventory of the superfamily of P-type ion pumps in Arabidopsis. Plant Physiol 126, 696-706. 10.1104/pp.126.2.696.

Baginski, E.S., Foa, P.P., and Zak, B. (1967). Microdetermination of inorganic phosphate, phospholipids, and total phosphate in biologic materials. Clin Chem 13, 326-332.

Barbour, R., Kling, K., Anderson, J.P., Banducci, K., Cole, T., Diep, L., Fox, M., Goldstein, J.M., Soriano, F., Seubert, P., and Chilcote, T.J. (2008). Red Blood Cells Are the Major Source of Alpha-Synuclein in Blood. Neurodegenerative Diseases 5, 55-59. 10.1159/000112832.

Berrocal, M., Corbacho, I., Sepulveda, M.R., Gutierrez-Merino, C., and Mata, A.M. (2017). Phospholipids and calmodulin modulate the inhibition of PMCA activity by tau. Biochim Biophys Acta Mol Cell Res 1864, 1028-1035. 10.1016/j.bbamcr.2016.10.023.

Betzer, C., Lassen, L.B., Olsen, A., Kofoed, R.H., Reimer, L., Gregersen, E., Zheng, J., Cali, T., Gai, W.P., Chen, T., et al. (2018). Alpha-synuclein aggregates activate calcium pump SERCA leading to calcium dysregulation. EMBO Rep 19. 10.15252/embr.201744617.

Betzer, C., Movius, A.J., Shi, M., Gai, W.P., Zhang, J., and Jensen, P.H. (2015). Identification of synaptosomal proteins binding to monomeric and oligomeric alpha-synuclein. PLoS One 10, e0116473. 10.1371/journal.pone.0116473.

Bodis-Wollner, I., Kozlowski, P.B., Glazman, S., and Miri, S. (2014). alpha-synuclein in the inner retina in parkinson disease. Ann Neurol 75, 964-966. 10.1002/ana.24182.

Bodner, C.R., Dobson, C.M., and Bax, A. (2009). Multiple Tight Phospholipid-Binding Modes of alpha-Synuclein Revealed by Solution NMR Spectroscopy. J Mol Biol 390, 775-790. 10.1016/j.jmb.2009.05.066. 
Bogdanova, A., Makhro, A., Wang, J., Lipp, P., and Kaestner, L. (2013). Calcium in red blood cells-a perilous balance. International journal of molecular sciences 14, 9848-9872. 10.3390/ijms14059848.

Brini, M., Di Leva, F., Ortega, C.K., Domi, T., Ottolini, D., Leonardi, E., Tosatto, S.C.E., and Carafoli, E. (2010). Deletions and Mutations in the Acidic Lipid-binding Region of the Plasma Membrane Ca2+ Pump: A STUDY ON DIFFERENT SPLICING VARIANTS OF ISOFORM 2. Journal of Biological Chemistry 285, 30779-30791. 10.1074/jbc.M110.140475.

Brodin, P., Falchetto, R., Vorherr, T., and Carafoli, E. (1992). Identification of two domains which mediate the binding of activating phospholipids to the plasma-membrane Ca2+ pump. Eur J Biochem 204, 939-946. 10.1111/j.1432-1033.1992.tb16715.x.

Burre, J., Sharma, M., Tsetsenis, T., Buchman, V., Etherton, M.R., and Sudhof, T.C. (2010). alpha-Synuclein Promotes SNARE-Complex Assembly in Vivo and in Vitro. Science 329, 16631667. 10.1126/science.1195227.

Cali, T., Brini, M., and Carafoli, E. (2017). Regulation of Cell Calcium and Role of Plasma Membrane Calcium ATPases. Int Rev Cell Mol Biol 332, 259-296. 10.1016/bs.ircmb.2017.01.002.

Cali, T., Brini, M., and Carafoli, E. (2018). The PMCA pumps in genetically determined neuronal pathologies. Neurosci Lett 663, 2-11. 10.1016/j.neulet.2017.11.005.

Cali, T., Ottolini, D., and Brini, M. (2014). Calcium signaling in Parkinson's disease. Cell Tissue Res 357, 439-454. 10.1007/s00441-014-1866-0.

Carafoli, E. (1994). Biogenesis: plasma membrane calcium ATPase: 15 years of work on the purified enzyme. The FASEB Journal 8, 993-1002.

Carafoli, E., and Stauffer, T. (1994). The plasma membrane calcium pump: functional domains, regulation of the activity, and tissue specificity of isoform expression. J Neurobiol 25, 312-324. 10.1002/neu.480250311.

Caride, A.J., Elwess, N.L., Verma, A.K., Filoteo, A.G., Enyedi, A., Bajzer, Z., and Penniston, J.T. (1999). The rate of activation by calmodulin of isoform 4 of the plasma membrane Ca2+ pump is slow and is changed by alternatives splicing. Journal of Biological Chemistry 274, 3522735232. 10.1074/jbc.274.49.35227.

Caride, A.J., Filoteo, A.G., Penniston, J.T., and Strehler, E.E. (2007). The plasma membrane $\mathrm{Ca} 2+$ pump isoform $4 \mathrm{a}$ differs from isoform $4 \mathrm{~b}$ in the mechanism of calmodulin binding and activation kinetics: implications for Ca2+ signaling. J Biol Chem 282, 25640-25648. 10.1074/jbc.M701129200.

Chen, H.Y., and Chesler, M. (2015). Autocrine boost of NMDAR current in hippocampal CA1 pyramidal neurons by a PMCA-dependent, perisynaptic, extracellular pH shift. J Neurosci 35, 873-877. 10.1523/JNEUROSCI.2293-14.2015.

Cheng, F., Vivacqua, G., and Yu, S. (2011). The role of alpha-synuclein in neurotransmission and synaptic plasticity. J Chem Neuroanat 42, 242-248. 10.1016/j.jchemneu.2010.12.001.

Cholak, E., Bugge, K., Khondker, A., Gauger, K., Pedraz-Cuesta, E., Pedersen, M.E., Bucciarelli, S., Vestergaard, B., Pedersen, S.F., Rheinstadter, M.C., et al. (2020). Avidity within the Nterminal anchor drives alpha-synuclein membrane interaction and insertion. FASEB J 34, 74627482. 10.1096/fj.202000107R.

Cunningham, K.W., and Fink, G.R. (1994). Calcineurin-dependent growth control in Saccharomyces cerevisiae mutants lacking PMC1, a homolog of plasma membrane Ca2+ ATPases. J Cell Biol 124, 351-363. 
Danzer, K.M., Haasen, D., Karow, A.R., Moussaud, S., Habeck, M., Giese, A., Kretzschmar, H., Hengerer, B., and Kostka, M. (2007). Different species of alpha-synuclein oligomers induce calcium influx and seeding. J Neurosci 27, 9220-9232. 10.1523/JNEUROSCI.2617-07.2007.

Davidson, W.S., Jonas, A., Clayton, D.F., and George, J.M. (1998). Stabilization of alphasynuclein secondary structure upon binding to synthetic membranes. J Biol Chem 273, 94439449. 10.1074/jbc.273.16.9443.

Di Leva, F., Domi, T., Fedrizzi, L., Lim, D., and Carafoli, E. (2008). The plasma membrane Ca2+ ATPase of animal cells: structure, function and regulation. Arch Biochem Biophys 476, 65-74. 10.1016/j.abb.2008.02.026.

Di Scala, C., Yahi, N., Boutemeur, S., Flores, A., Rodriguez, L., Chahinian, H., and Fantini, J. (2016). Common molecular mechanism of amyloid pore formation by Alzheimer's betaamyloid peptide and alpha-synuclein. Scientific Reports 6. ARTN 28781 10.1038/srep28781.

Dikiy, I., and Eliezer, D. (2012). Folding and misfolding of alpha-synuclein on membranes. BbaBiomembranes 1818, 1013-1018. 10.1016/j.bbamem.2011.09.008.

Domi, T., Di Leva, F., Fedrizzi, L., Rimessi, A., and Brini, M. (2007). Functional specificity of PMCA isoforms? Ann N Y Acad Sci 1099, 237-246. 10.1196/annals.1387.043.

Drew, D., Newstead, S., Sonoda, Y., Kim, H., von Heijne, G., and Iwata, S. (2008). GFP-based optimization scheme for the overexpression and purification of eukaryotic membrane proteins in Saccharomyces cerevisiae. Nat Protoc 3, 784-798. 10.1038/nprot.2008.44.

Eliezer, D., Kutluay, E., Bussell, R., Jr., and Browne, G. (2001). Conformational properties of alpha-synuclein in its free and lipid-associated states. J Mol Biol 307, 1061-1073. 10.1006/jmbi.2001.4538.

Enyedi, A., and Strehler, E.E. (2011). Regulation of apical membrane enrichment and retention of plasma membrane Ca ATPase splice variants by the PDZ-domain protein NHERF2. Commun Integr Biol 4, 340-343. 10.4161/cib.4.3.15040.

Fanning, S., Selkoe, D., and Dettmer, U. (2020). Parkinson's disease: proteinopathy or lipidopathy? NPJ Parkinsons Dis 6, 3. 10.1038/s41531-019-0103-7.

Fauvet, B., Mbefo, M.K., Fares, M.B., Desobry, C., Michael, S., Ardah, M.T., Tsika, E., Coune, P., Prudent, M., Lion, N., et al. (2012). alpha-Synuclein in central nervous system and from erythrocytes, mammalian cells, and Escherichia coli exists predominantly as disordered monomer. J Biol Chem 287, 15345-15364. 10.1074/jbc.M111.318949.

Feghhi, T., Hernandez, R.X., Stawarski, M., Thomas, C.I., Kamasawa, N., Lau, A.W.C., and Macleod, G.T. (2021). Computational modeling predicts ephemeral acidic microdomains in the glutamatergic synaptic cleft. Biophys J 120, 5575-5591. 10.1016/j.bpj.2021.11.011.

Gietz, R.D., and Schiestl, R.H. (2007). High-efficiency yeast transformation using the LiAc/SS carrier DNA/PEG method. Nat Protoc 2, 31-34. 10.1038/nprot.2007.13.

Gong, D., Chi, X., Ren, K., Huang, G., Zhou, G., Yan, N., Lei, J., and Zhou, Q. (2018). Structure of the human plasma membrane $\mathrm{Ca}(2+)$-ATPase 1 in complex with its obligatory subunit neuroplastin. Nat Commun 9, 3623. 10.1038/s41467-018-06075-7.

Habeck, M., Kapri-Pardes, E., Sharon, M., and Karlish, S.J. (2017). Specific phospholipid binding to Na,K-ATPase at two distinct sites. Proc Natl Acad Sci U S A 114, 2904-2909. 10.1073/pnas.1620799114.

Huang, C., Ren, G., Zhou, H., and Wang, C.C. (2005). A new method for purification of recombinant human alpha-synuclein in Escherichia coli. Protein Expr Purif 42, 173-177. 10.1016/j.pep.2005.02.014. 
Hurley, M.J., Brandon, B., Gentleman, S.M., and Dexter, D.T. (2013). Parkinson's disease is associated with altered expression of $\mathrm{Ca}(\mathrm{V}) 1$ channels and calcium-binding proteins. Brain 136, 2077-2097. 10.1093/brain/awt134.

Hurley, M.J., Gentleman, S.M., and Dexter, D.T. (2015). Calcium CaV1 channel subtype mRNA expression in Parkinson's disease examined by in situ hybridization. J Mol Neurosci 55, 715724. 10.1007/s12031-014-0410-8.

Jensen, P.H., Nielsen, M.S., Jakes, R., Dotti, G., and Goedert, M. (1998). Binding of alphasynuclein to brain vesicles is abolished by familial Parkinson's disease mutation. Journal of Biological Chemistry 273, 26292-26294. DOI 10.1074/jbc.273.41.26292.

Jo, E., Fuller, N., Rand, R.P., St George-Hyslop, P., and Fraser, P.E. (2002). Defective membrane interactions of familial Parkinson's disease mutant A30P alpha-synuclein. J Mol Biol 315, 799807. 10.1006/jmbi.2001.5269.

Jo, E.J., McLaurin, J., Yip, C.M., St George-Hyslop, P., and Fraser, P.E. (2000). alpha-synuclein membrane interactions and lipid specificity. Journal of Biological Chemistry 275, 34328-34334. DOI 10.1074/jbc.M004345200.

Kessler, F., Falchetto, R., Heim, R., Meili, R., Vorherr, T., Strehler, E.E., and Carafoli, E. (1992). Study of calmodulin binding to the alternatively spliced C-terminal domain of the plasma membrane Ca2+ pump. Biochemistry 31, 11785-11792.

Kragh, C.L., Lund, L.B., Febbraro, F., Hansen, H.D., Gai, W.-P., El-Agnaf, O., Richter-Landsberg, C., and Jensen, P.H. (2009). $\alpha$-Synuclein Aggregation and Ser-129 Phosphorylation-dependent Cell Death in Oligodendroglial Cells. Journal of Biological Chemistry 284, 10211-10222. 10.1074/jbc.M809671200.

Krebs, J. (2015). The plethora of PMCA isoforms: Alternative splicing and differential expression. Biochim Biophys Acta 1853, 2018-2024. 10.1016/j.bbamcr.2014.12.020.

Krick, N., Ryglewski, S., Pichler, A., Bikbaev, A., Gotz, T., Kobler, O., Heine, M., Thomas, U., and Duch, C. (2021). Separation of presynaptic Cav2 and Cav1 channel function in synaptic vesicle exo- and endocytosis by the membrane anchored $\mathrm{Ca}(2+)$ pump PMCA. Proc Natl Acad Sci U S A 118. 10.1073/pnas.2106621118.

Krizaj, D., Demarco, S.J., Johnson, J., Strehler, E.E., and Copenhagen, D.R. (2002). Cell-specific expression of plasma membrane calcium ATPase isoforms in retinal neurons. J Comp Neurol 451, 1-21. 10.1002/cne.10281.

Kruger, R., Kuhn, W., Leenders, K.L., Sprengelmeyer, R., Muller, T., Woitalla, D., Portman, A.T., Maguire, R.P., Veenma, L., Schroder, U., et al. (2001). Familial parkinsonism with synuclein pathology: clinical and PET studies of A30P mutation carriers. Neurology 56, 1355-1362. 10.1212/wnl.56.10.1355.

Lautenschlager, J., Stephens, A.D., Fusco, G., Strohl, F., Curry, N., Zacharopoulou, M., Michel, C.H., Laine, R., Nespovitaya, N., Fantham, M., et al. (2018). C-terminal calcium binding of alpha-synuclein modulates synaptic vesicle interaction. Nat Commun 9, 712. 10.1038/s41467018-03111-4.

Lindersson, E., Beedholm, R., Højrup, P., Moos, T., Gai, W., Hendil, K.B., and Jensen, P.H. (2004). Proteasomal Inhibition by $\alpha$-Synuclein Filaments and Oligomers. Journal of Biological Chemistry 279, 12924-12934. 10.1074/jbc.M306390200.

Liu, H., and Naismith, J.H. (2008). An efficient one-step site-directed deletion, insertion, single and multiple-site plasmid mutagenesis protocol. BMC Biotechnol 8, 91. 10.1186/1472-67508-91.

Long, A.A., Kim, E., Leung, H.T., Woodruff, E., 3rd, An, L., Doerge, R.W., Pak, W.L., and Broadie, K. (2008). Presynaptic calcium channel localization and calcium-dependent synaptic vesicle 
exocytosis regulated by the Fuseless protein. J Neurosci 28, 3668-3682. 10.1523/JNEUROSCI.5553-07.2008.

Lopreiato, R., Giacomello, M., and Carafoli, E. (2014). The plasma membrane calcium pump: new ways to look at an old enzyme. J Biol Chem 289, 10261-10268. 10.1074/jbc.0114.555565. Nielsen, M.S., Vorum, H., Lindersson, E., and Jensen, P.H. (2001). Ca2+ binding to alphasynuclein regulates ligand binding and oligomerization. J Biol Chem 276, 22680-22684. 10.1074/jbc.M101181200.

Niggli, V., Adunyah, E.S., and Carafoli, E. (1981a). Acidic phospholipids, unsaturated fatty acids, and limited proteolysis mimic the effect of calmodulin on the purified erythrocyte Ca2+ ATPase. J Biol Chem 256, 8588-8592.

Niggli, V., Adunyah, E.S., Penniston, J.T., and Carafoli, E. (1981b). Purified (Ca2+-Mg2+)-ATPase of the erythrocyte membrane. Reconstitution and effect of calmodulin and phospholipids. J Biol Chem 256, 395-401.

Pei, Y., and Maitta, R.W. (2019). Alpha synuclein in hematopoiesis and immunity. Heliyon 5, e02590. 10.1016/j.heliyon.2019.e02590.

Penniston, J.T., Padanyi, R., Paszty, K., Varga, K., Hegedus, L., and Enyedi, A. (2014). Apart from its known function, the plasma membrane $\mathrm{Ca}(2)(+)$ ATPase can regulate $\mathrm{Ca}(2)(+)$ signaling by controlling phosphatidylinositol 4,5-bisphosphate levels. J Cell Sci 127, 72-84. 10.1242/jcs.132548.

Perni, M., Galvagnion, C., Maltsev, A., Meisl, G., Muller, M.B.D., Challa, P.K., Kirkegaard, J.B., Flagmeier, P., Cohen, S.I.A., Cascella, R., et al. (2017). A natural product inhibits the initiation of alpha-synuclein aggregation and suppresses its toxicity (vol 114, pg E1009, 2017). P Natl Acad Sci USA 114, E2543-E2543. 10.1073/pnas.1701964114.

Pinto Fde, T., and Adamo, H.P. (2002). Deletions in the acidic lipid-binding region of the plasma membrane $\mathrm{Ca} 2+$ pump. A mutant with high affinity for $\mathrm{Ca} 2+$ resembling the acidic lipidactivated enzyme. The Journal of biological chemistry 277, 12784-12789. 10.1074/jbc.M111055200.

Rcom-H'cheo-Gauthier, A.N., Osborne, S.L., Meedeniya, A.C., and Pountney, D.L. (2016). Calcium: Alpha-Synuclein Interactions in Alpha-Synucleinopathies. Front Neurosci 10, 570. 10.3389/fnins.2016.00570.

Singh, N., Bartol, T., Levine, H., Sejnowski, T., and Nadkarni, S. (2021). Presynaptic endoplasmic reticulum regulates short-term plasticity in hippocampal synapses. Commun Biol 4, 241. 10.1038/s42003-021-01761-7.

Sorensen, T.L., Clausen, J.D., Jensen, A.M., Vilsen, B., Moller, J.V., Andersen, J.P., and Nissen, $\mathrm{P}$. (2004). Localization of a $\mathrm{K}+-$-binding site involved in dephosphorylation of the sarcoplasmic reticulum Ca2+ -ATPase. J Biol Chem 279, 46355-46358. 10.1074/jbc.C400414200.

Strehler, E.E. (2015). Plasma membrane calcium ATPases: From generic Ca2+ sump pumps to versatile systems for fine-tuning cellular $\mathrm{Ca} 2+$. Biochemical and Biophysical Research Communications 460, 26-33. https://doi.org/10.1016/j.bbrc.2015.01.121.

Strehler, E.E., Caride, A.J., Filoteo, A.G., Xiong, Y., Penniston, J.T., and Enyedi, A. (2007a). Plasma membrane Ca2+ ATPases as dynamic regulators of cellular calcium handling. Ann N Y Acad Sci 1099, 226-236. 10.1196/annals.1387.023.

Strehler, E.E., Filoteo, A.G., Penniston, J.T., and Caride, A.J. (2007b). Plasma-membrane Ca(2+) pumps: structural diversity as the basis for functional versatility. Biochem Soc Trans 35, 919922. 10.1042/bst0350919. 
Strehler, E.E., and Thayer, S.A. (2018). Evidence for a role of plasma membrane calcium pumps in neurodegenerative disease: Recent developments. Neurosci Lett 663, 39-47. 10.1016/j.neulet.2017.08.035.

Strehler, E.E., and Zacharias, D.A. (2001). Role of Alternative Splicing in Generating Isoform Diversity Among Plasma Membrane Calcium Pumps. Physiological Reviews 81, 21-50.

Sulzer, D., and Edwards, R.H. (2019). The physiological role of alpha-synuclein and its relationship to Parkinson's Disease. J Neurochem 150, 475-486. 10.1111/jnc.14810.

Theillet, F.X., Binolfi, A., Bekei, B., Martorana, A., Rose, H.M., Stuiver, M., Verzini, S., Lorenz, D., van Rossum, M., Goldfarb, D., and Selenko, P. (2016). Structural disorder of monomeric alpha-synuclein persists in mammalian cells. Nature 530, 45-+. 10.1038/nature16531.

Tidow, H., Poulsen, L.R., Andreeva, A., Knudsen, M., Hein, K.L., Wiuf, C., Palmgren, M.G., and Nissen, P. (2012). A bimodular mechanism of calcium control in eukaryotes. Nature 491, 468472. 10.1038/nature11539.

Ulmer, T.S., Bax, A., Cole, N.B., and Nussbaum, R.L. (2005). Structure and dynamics of micellebound human alpha-synuclein. J Biol Chem 280, 9595-9603. 10.1074/jbc.M411805200.

Uversky, V.N., and Eliezer, D. (2009). Biophysics of Parkinson's disease: structure and aggregation of alpha-synuclein. Curr Protein Pept Sci 10, 483-499. $10.2174 / 138920309789351921$.

Vargas, K.J., Schrod, N., Davis, T., Fernandez-Busnadiego, R., Taguchi, Y.V., Laugks, U., Lucic, V., and Chandra, S.S. (2017). Synucleins Have Multiple Effects on Presynaptic Architecture. Cell Rep 18, 161-173. 10.1016/j.celrep.2016.12.023.

Wu, Y., Whiteus, C., Xu, C.S., Hayworth, K.J., Weinberg, R.J., Hess, H.F., and De Camilli, P. (2017). Contacts between the endoplasmic reticulum and other membranes in neurons. Proc Natl Acad Sci U S A 114, E4859-E4867. 10.1073/pnas.1701078114.

Zvaritch, E., James, P., Vorherr, T., Falchetto, R., Modyanov, N., and Carafoli, E. (1990). Mapping of functional domains in the plasma membrane Ca2+ pump using trypsin proteolysis. Biochemistry 29, 8070-8076. 10.1021/bi00487a012. 
Supplemental information

\section{Stimulation of Plasma Membrane Calcium Pumps by Monomeric $\alpha-S y n u c l e i n$}

Antoni Kowalski, Cristine Betzer, Sigrid Thirup Larsen, Emil Gregersen, Montaña Caballero

Bermejo, Poul Henning Jensen, Poul Nissen 
Figure S1
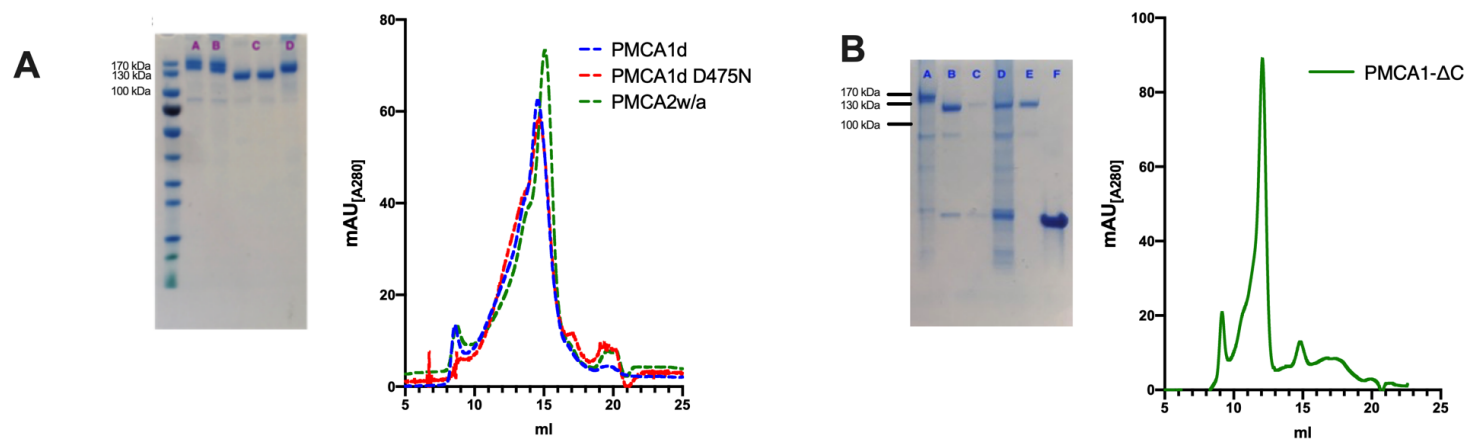

C
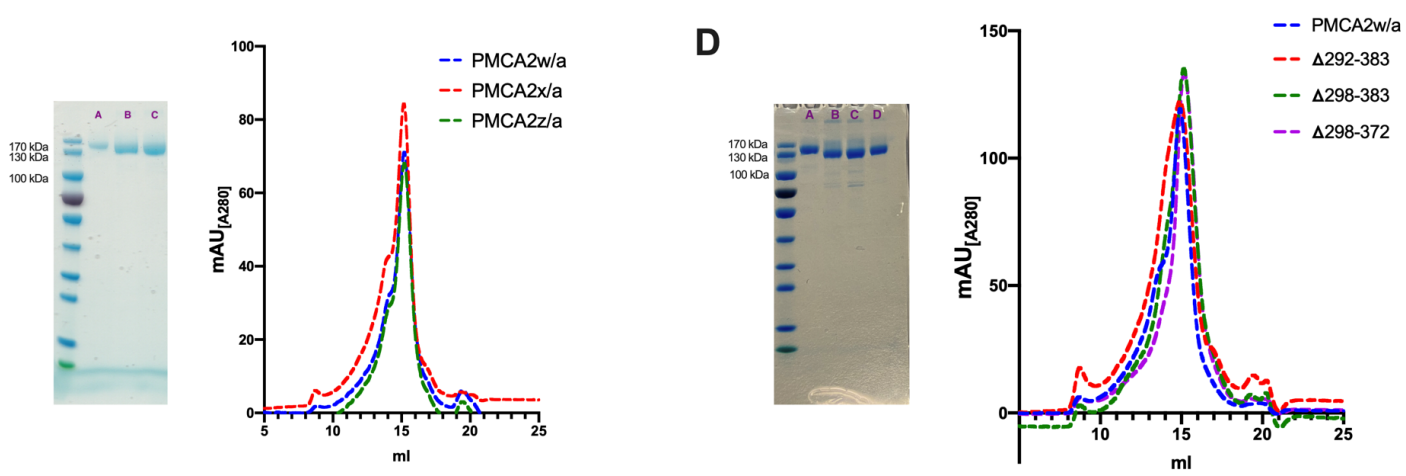
Figure S2

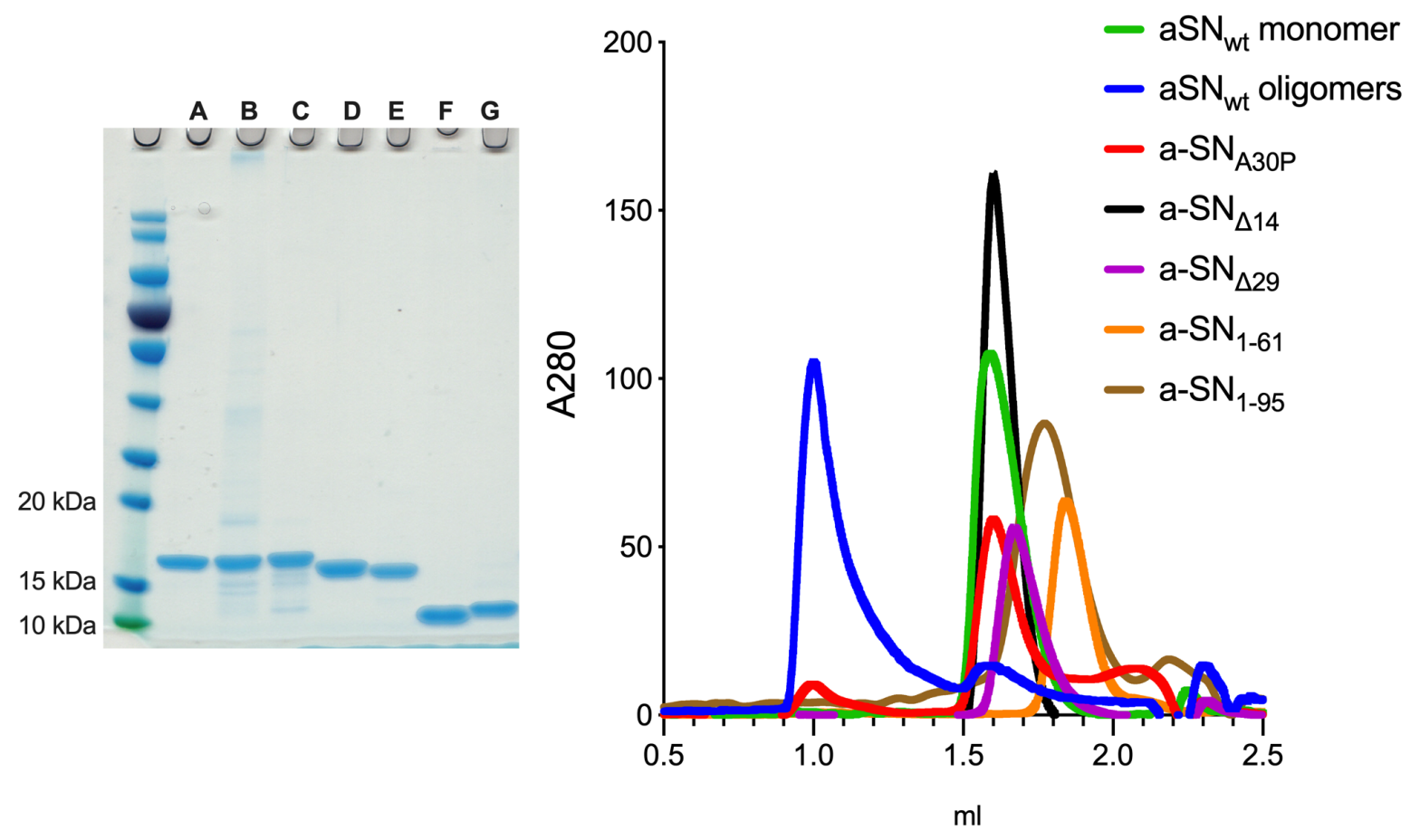


Figure S3

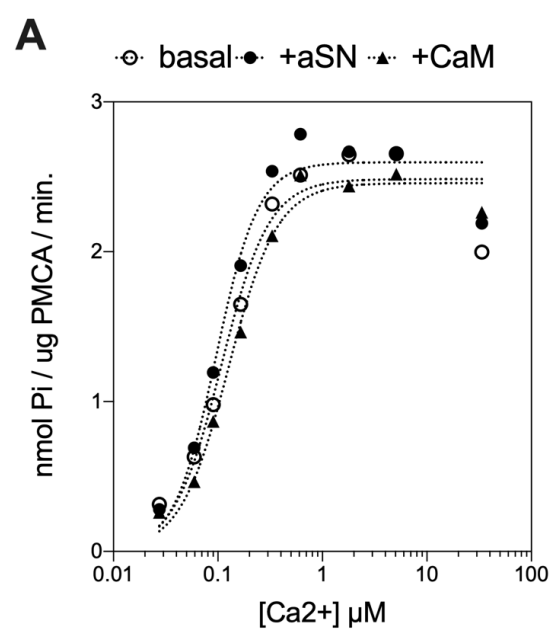

B

Figure S4

A-TM3 linker region

TM3

PMCA2D298-372 273 - VTAVGVNSQTGIIFTLLGAGGEEEERKKASMHKKEKSVLQGKLTKLAVQIGKAGLVMSAI-TV

PMCA2D298-383 273 - VTAVGVNSQTGIIFTLLGAGGEEEE---------SVLQGKLTKLAVQIGKAGLVMSAI-TV

ACA8 339 - VTGVGVNTEWGLLMASISEDNGEE-.-.-.-.-TPLQVRLNGVATFIGSIGLAVAAAVLV

SERCA1a 223 - VATTGVSTEIGKIRDQMAAT--------EQDKTPLQQKLDEFGEQLSKVISLICVAVWL 
The original code written in $\mathrm{R}$ for the purpose of the analysis of transcriptomics data. Remaining data reported in this paper will be shared by the lead contact upon request.

The code relevant for the analysis displayed on the Figure 3A:

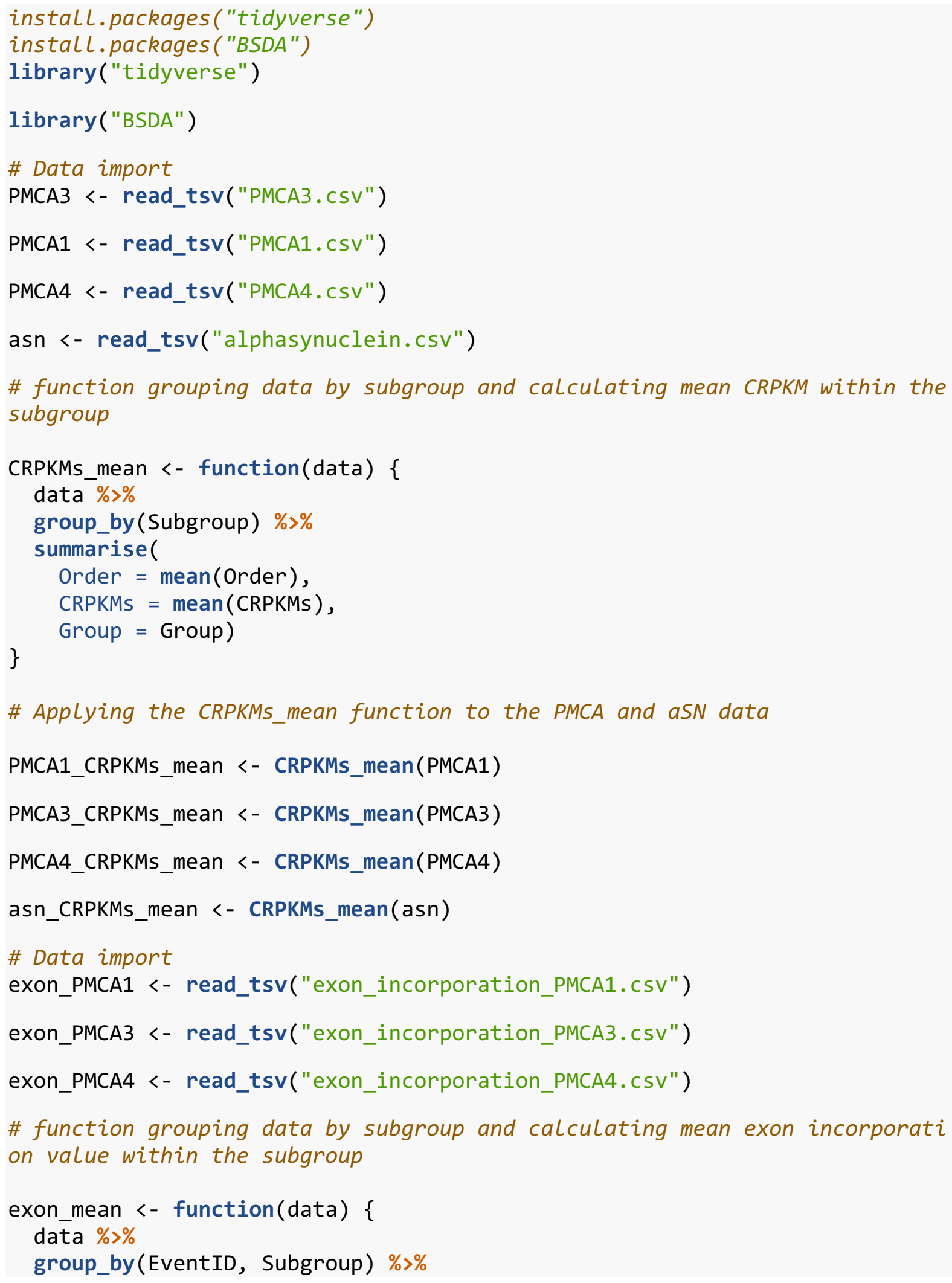




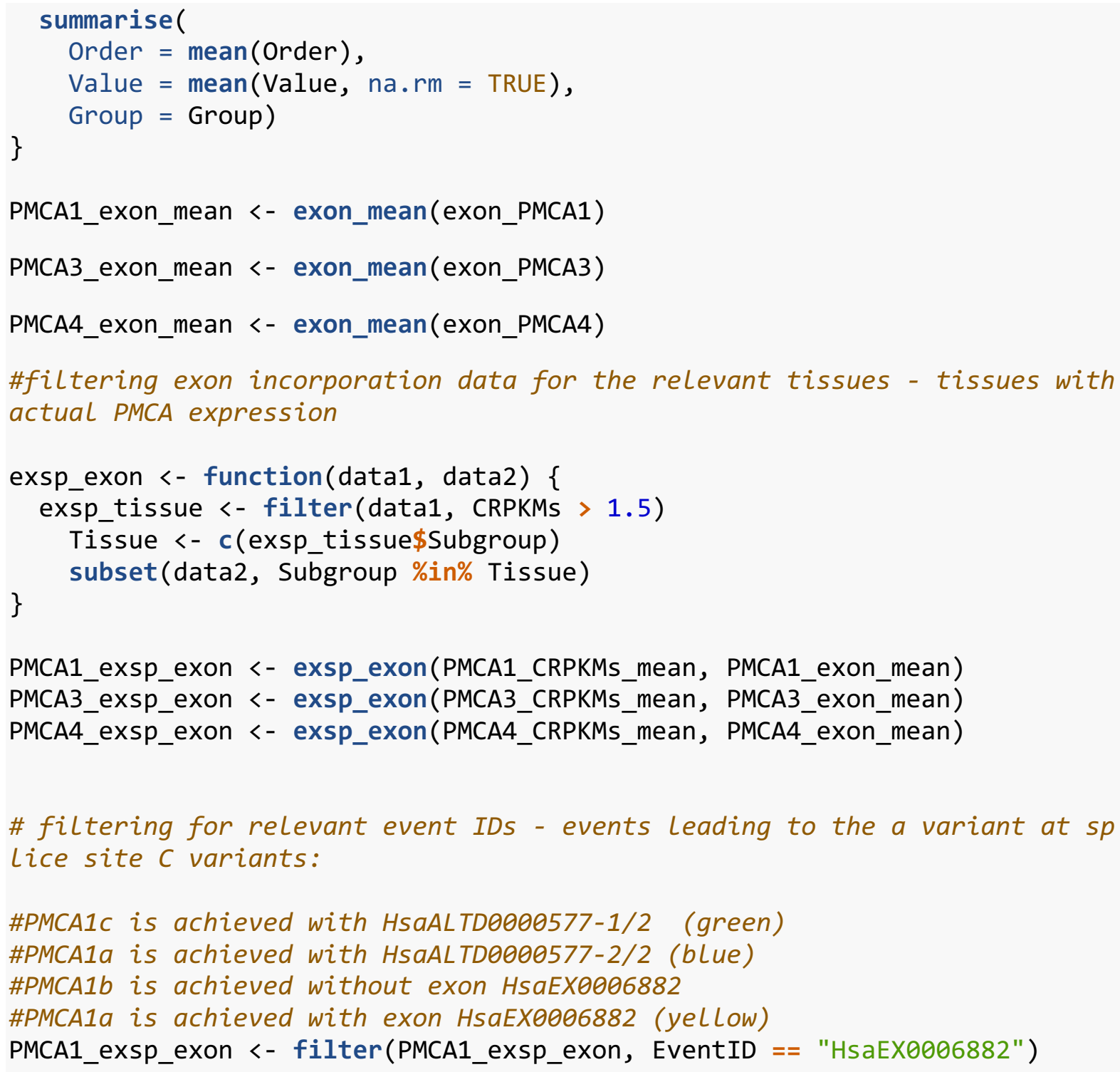

\# filtering for relevant event IDs - events leading to the a variant at $s p$ Lice site $C$ variants:

\#PMCA1c is achieved with HsaALTD0000577-1/2 (green)

\#PMCA1a is achieved with HsaALTD0000577-2/2 (blue)

\#PMCA1b is achieved without exon HsaEX0006882

\#PMCA1a is achieved with exon HsaEX0006882 (yellow)

PMCA1_exsp_exon <- filter(PMCA1_exsp_exon, EventID == "HsaEX0006882")

\#HsaEX0006895 with incorporation PMCA3x without PMCA3z, HsaEX0006897 with then PMCA3a and without then PMCA3b

PMCA3_exsp_exon <- filter(PMCA3_exsp_exon, EventID == "HsaEX0006897")

\#PMCA4Z is achieved without the HsaEX0006899 exon \#PMCA4x is achieved with the HsaEX0006899 exon \#PMCA4a is achieved with the HsaEX0006904 exon \#PMCA $4 b$ is achieved without the HsaEX0006904 exon

PMCA4_exsp_exon <- filter(PMCA4_exsp_exon, EventID == "HsaEX0006904")

\# function for calculation of mean incorporation of a specific splicing ev ent for tissues within a designated interval of aSN expression

Event_mean_int_PMCA $<-$ function(PMCA_data) \{

interval <- $\operatorname{seq}(0,190$, by=10)

exsp_asn <- list ()

Event_mean_PMCA <- list()

Event_mean_all_tissues <- list() 


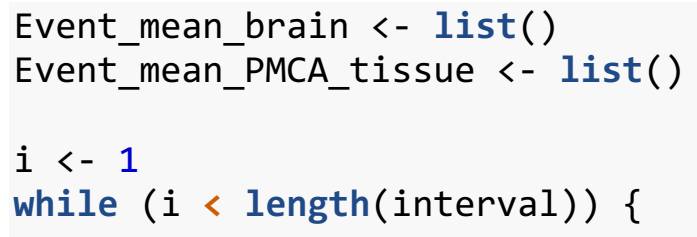

exsp_asn[[paste(interval[[i+1]])]] <- filter(asn_CRPKMs_mean, CRPKMs >= interval[[i]] \& CRPKMs < interval[[i+1]])

Event_mean_PMCA[[paste(interval[[i+1]])]] <- subset(PMCA_data, Subgroup \%in\% exsp_asn[[i]]\$Subgroup)

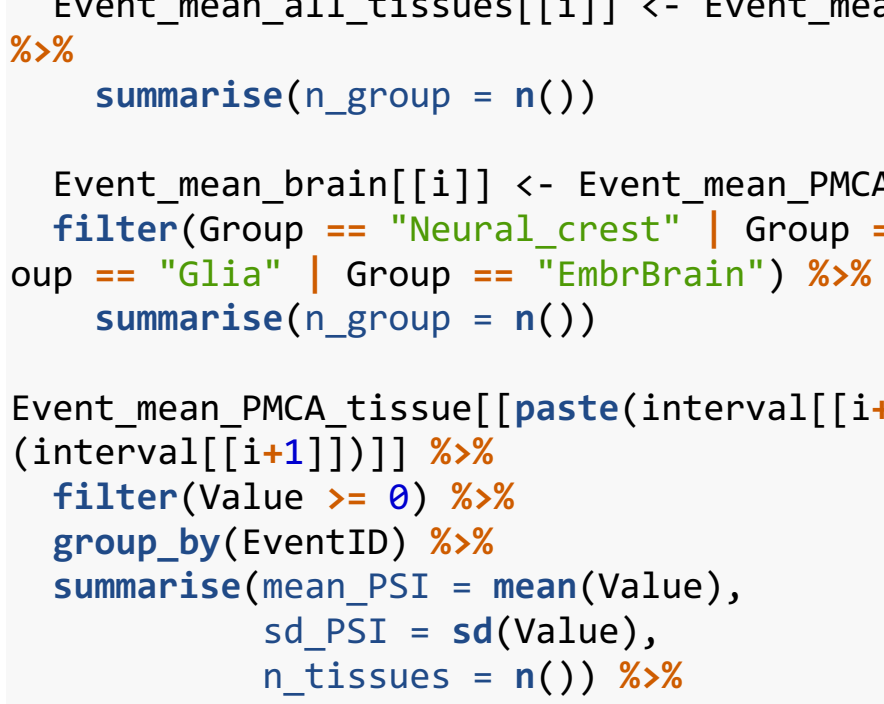

add_column(percent_brain = sum(Event_mean_brain[[i]]\$n_group)/sum(Event_ mean_all_tissues[[i]] \$n_group)*100) \%>\% 
PMCA1_a <- Event_mean_int_PMCA1

PMCA3_a <- Event_mean_int_PMCA3

PMCA4_a <- Event_mean_int_PMCA4

\# The tissues within aSN intervals 110-120 and 130-140. These are outliers

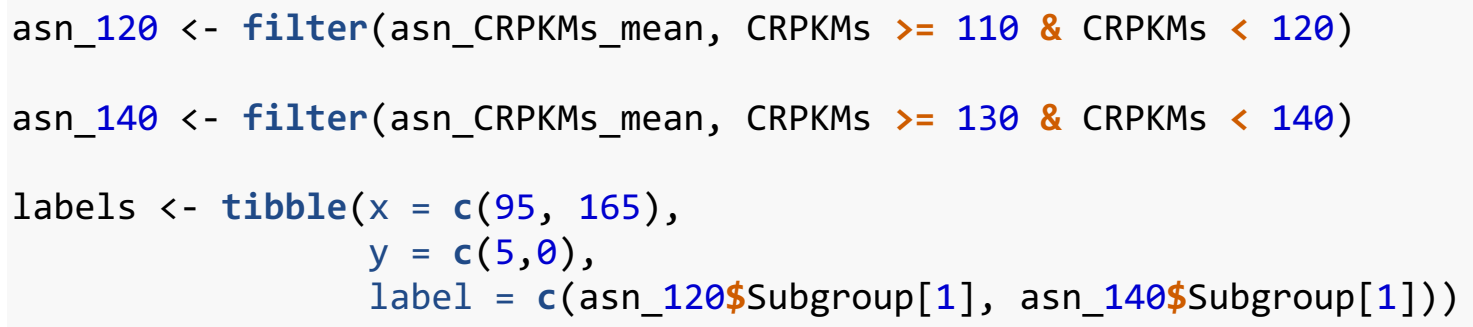

\# Plotting mean incorporation of events leading to variant a at different aSN intervals

ggplot ( ) +

geom_point $($ data $=$ PMCA1_a, mapping $=$ aes $(x=$ asn_exp_interval, $y=$ mean PSI, color $=$ EventID), size $=2.5)+$

geom_point $($ data $=$ PMCA4_a, mapping $=$ aes $(x=$ asn_exp_interval, $y=$ mean PSI, color $=$ EventID), size $=2.5)+$

geom_point (data $=$ PMCA3_a, mapping $=$ aes $(x=$ asn_exp_interval, $y=$ mean PSI, color $=$ EventID), size $=2.5)+$

theme_bw( ) +

theme (legend.title=element_blank ()$)+$

scale_color_manual(labels = c("PMCA1 \n HsaEX0006882", "PMCA3 \n HsaEX00 06897", "PMCA4 \n HsaEX0006904"), values=c("\#FFCC33", "\#9900CC", "\#0000FF") ) + ") +

labs $(x=$ "aSN expression interval (CRPKMs)", $y=$ "mean PSI \n PMCA exons theme (axis.text. $x=$ element_text $($ angle $=90$, hjust $=1$, vjust $=0.5))+$ theme(legend.position="bottom") +

scale_x_continuous $($ breaks $=\operatorname{seq}(10,190$, by $=10)$, labels $=$ expression_i nterval) + )+

geom_label $($ data $=$ labels, mapping $=\operatorname{aes}(x=x, y=y, l a b e l=l a b e l)$, size $=3$

ggsave(width $=5$, height $=4$, filename $=$ "mean_PSI_interval_PMCA_a_versi on.png")

\# Plotting with standard deviations

ggplot () +

geom_point (data $=$ PMCA1_a, mapping $=$ aes $(x=$ asn_exp_interval, $y=$ mean_ PSI, color $=$ EventID $)$, size $=2.5)+$

geom_point (data $=$ PMCA4_a, mapping $=$ aes $(x=$ asn_exp_interval, $y=$ mean_

PSI, color $=$ EventID), size $=2.5)+$

geom_point (data $=$ PMCA3_a, mapping $=$ aes $(x=$ asn_exp_interval, $y=$ mean PSI, color $=$ EventID), size $=2.5)+$

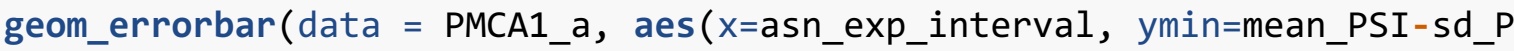
SI, ymax=mean_PSI+Sd_PSI, width $=5)$ ) +

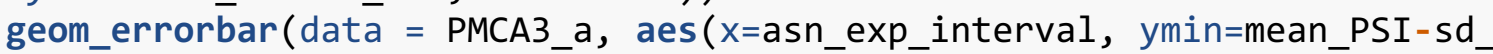
PSI, $y$ max $=$ mean_PSI+sd_PSI, width $=5)$ )+ 
geom_errorbar (data = PMCA4_a, aes (x=asn_exp_interval, ymin=mean_PSI-sd_ PSI, ymax=mean_PSI+Sd_PSI, width $=5)$ )+

theme_bw( ) +

theme (axis.text. $x=$ element_text $($ angle $=90$, hjust $=1$, vjust $=0.5))+$ theme(legend.title=element_blank()) +

scale_color_manual(labels = c("PMCA1 \n HsaEX0006882", "PMCA3 \n HsaEX00 06897", "PMCA4 \n HsaEX0006904"), values=c("\#FFCC33","\#9900CC", "\#0000FF") ) +

labs $(x=$ "aSN expression interval (CRPKMs)", $y=$ "mean PSI \n PMCA exons ") +

theme (axis.text.x $=$ element_text $($ angle $=90$, hjust $=1$, vjust $=0.5))+$ theme(legend. position="bottom") +

scale_x_continuous (breaks $=\operatorname{seq}(10,190$, by $=10)$, labels $=$ expression interval $)+$

geom_label(data $=$ labels, mapping $=\operatorname{aes}(x=x, y=y, l a b e l=l a b e l)$, size $=3$ )+

ggsave $($ width $=5$, height $=4$, filename $=$ "mean_PSI_interval_PMCA_a_ver sion_error.png")

\#t-test - calculate if there is a stastically significant difference betwe en the mean PSI for tissues with aSN expression below 50 CRPKMs and above 50 CRPKMS.

interval_means <- function(PMCA_data, interval1, interval2) \{

exsp_asn <- filter(asn_CRPKMs_mean, CRPKMs >= interval1 \& CRPKMs < interva 12) $\%$

Event_mean_PMCA <- subset(PMCA_data, Subgroup \%in\% exsp_asn\$Subgroup) \%>

group_by(EventID) \% \%

summarise(mean_PSI $=\operatorname{mean}($ Value $)$, sd_PSI = sd(Value),

\}

n_tissues $=\mathrm{n}()$ )

\#- For PMCA4

low_data_means <- interval_means(PMCA4_exsp_exon, 0, 50)

high_data_means <- interval_means(PMCA4_exsp_exon, 50, 190)

tsum.test (mean.x $=$ (low_data_means $[[1,2]]), \mathrm{s} \cdot \mathrm{x}=$ (low_data_means $[[1,3]])$, $\mathrm{n} \cdot \mathrm{x}=$ (low_data_means $[[\overline{1}, 4]])$, mean.y $=$ (high_data_means $[[1,2]]), \mathrm{s} \cdot \mathrm{y}=(\mathrm{h}$ igh_data_means $[\overline{[}[1,3]]), \mathrm{n} \cdot \mathrm{y}=($ high_data_means $[[1, \overline{4}]])$ )

\#\#

\#\# Welch Modified Two-Sample t-Test

\#\#

\#\# data: Summarized $\mathrm{x}$ and $\mathrm{y}$

$\# \#=-2.6275, \mathrm{df}=10.859, \mathrm{p}$-value $=0.02374$

\#\# alternative hypothesis: true difference in means is not equal to 0

\#\# 95 percent confidence interval:

\#\# $\quad-53.460998 \quad-4.680534$

\#\# sample estimates:

\#\# mean of $x$ mean of $y$

\#\# $16.24105 \quad 45.31182$ 
\#for PMCA1

low_data_means_1 <- interval_means(PMCA1_exsp_exon, 0, 50)

high_data_means_1 <- interval_means(PMCA1_exsp_exon, 50, 190)

tsum.test (mean.x $=$ (low_data_means_1 $[[1,2]]$ ), s.x $=$ (low_data_means_1 $[[1,3$

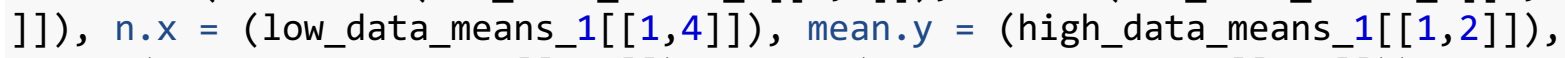
$\mathrm{s} \cdot \mathrm{y}=($ high_data_means_1 $[[\overline{1}, 3]]), \mathrm{n} \cdot \mathrm{y}=($ high_data_means_$\overline{1}[[1,4] \overline{]})$ )

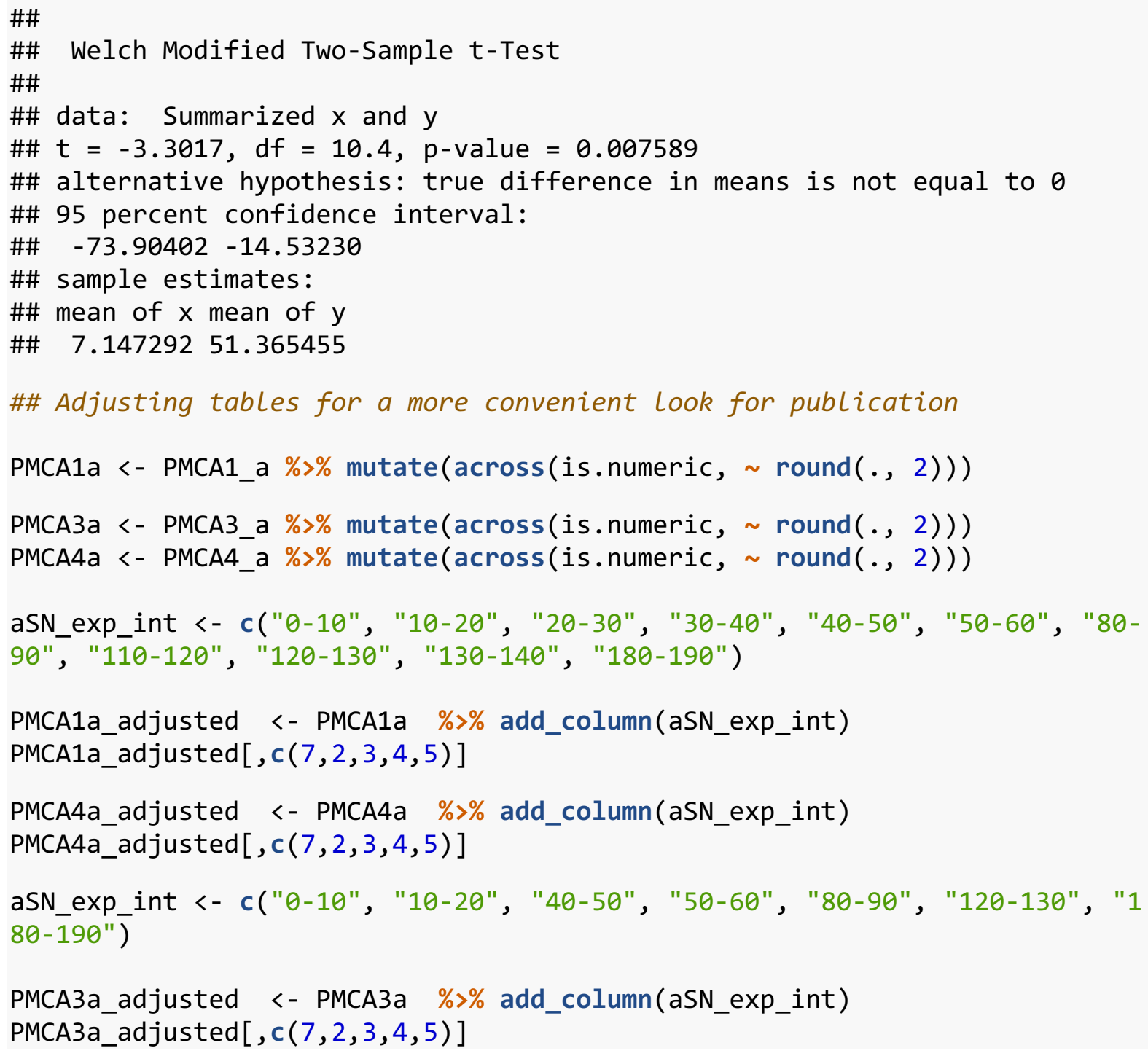


The code relevant for the analysis displayed on the Figure 3B:

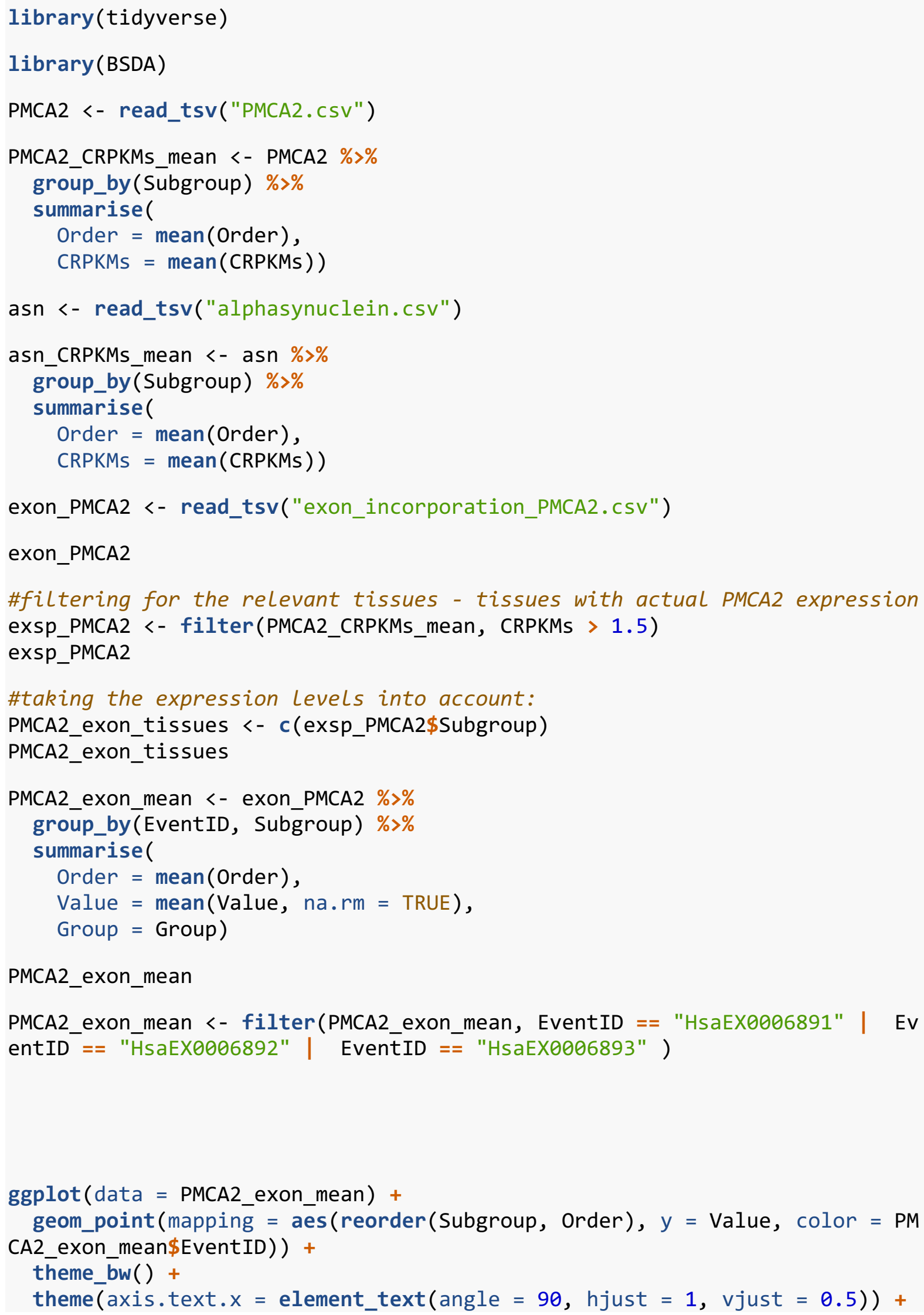


theme (legend.title=element_blank()) +

scale_color_manual(values=c("\#FF6600", "\#FFCC33", "\#000066"))

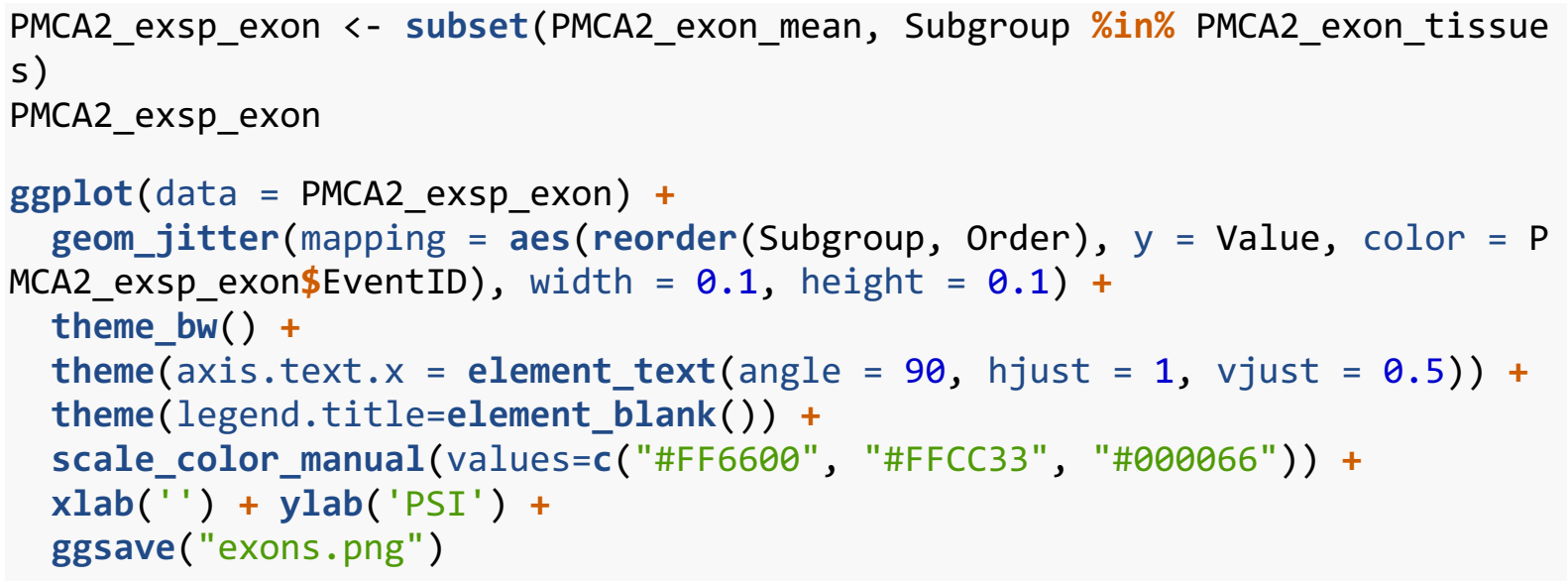

\#-_... The mean incorporation values for all tis

sues \#\#\#

exsp_asn <- filter(asn_CRPKMs_mean, CRPKMs > 0 )

Event_mean_PMCA2 <- subset(PMCA2_exsp_exon, Subgroup \%in\% exsp_asn $\$ S u b g r o u$ p) $\%>\%$

group_by(EventID) \%>\%

summarise (mean_PSI $=$ mean (Value), sd_PSI $=$ sd(Value $)$ )

Event_mean_PMCA2

expression_interval <- c("0-10", "10-20", "20-30", "30-40", "40-50", "50-6 0", "60-70", "70-80", "80-90", "90-100", "100-110", "110-120", "120-130", "130-140", "140-150", "150-160", "160-170", "170-180", "180-190")

\# function for calculation of mean incorporation of a specific splicing ev ent for tissues within a designated interval of aSN expression

Event_mean_int_PMCA <- function(PMCA_data) \{

interval <- $\operatorname{seq}(0,190$, by=10)

exsp_asn <- list ()

Event_mean_PMCA <- list()

Event_mean_all_tissues <- list()

Event_mean_brain <- list()

Event_mean_PMCA_tissue $<-$ list ()

$i<-1$

while $(i<$ length $($ interval $))\{$

exsp_asn[[paste(interval[[i+1]])]] <- filter(asn_CRPKMs_mean, CRPKMs >= interval[[i]] \& CRPKMs < interval[[i+1]])

Event_mean_PMCA $[$ paste $($ interval $[[i+1]])]]<-$ subset(PMCA_data, Subgroup 


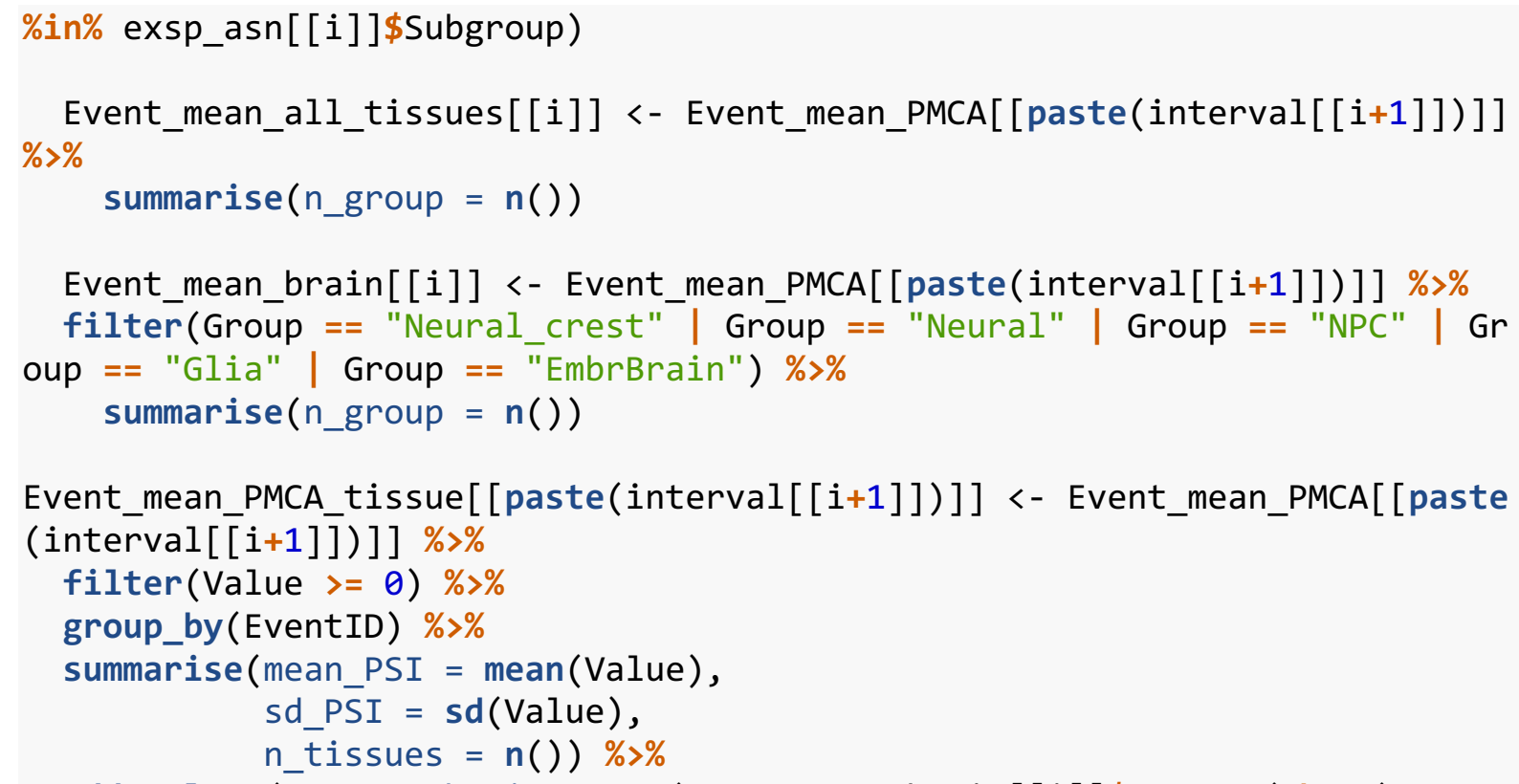

Event_mean_brain[[i]] <- Event_mean_PMCA[[paste(interval[[i+1]])]] \%>\%

filter(Group == "Neural_crest" | Group == "Neural" | Group == "NPC" | Gr oup == "Glia"| Group == "EmbrBrain") \% \%

summarise $\left(n \_g r o u p=n()\right)$

add_column (percent_brain = sum(Event_mean_brain[[i]]\$n_group)/sum(Event_ mean_all_tissues[[i]] $\$$ n_group) $* 100) \%>\overline{\%}$

add_column(asn_exp_interval = interval[[i+1]])

$i<-i+1$

\}

Event_mean_PMCA_tissue <- data.table::rbindlist(Event_mean_PMCA_tissue)

\}

Event_mean_int_PMCA2 <- Event_mean_int_PMCA(PMCA2_exsp_exon)

Event_mean_int_PMCA2

Event_mean_int_PMCA2 \% \%

$\operatorname{ggp} \bar{l}$ ot ()$^{-}+$

geom_point(mapping $=$ aes $(x=$ asn_exp_interval, $y=$ mean_PSI, color $=$ Eve ntID), size $=2.5)+$

geom_errorbar (aes (x=asn_exp_interval, ymin=mean_PSI-sd_PSI, ymax=mean_PS

I+sd_PSI, width $=5)$ ) +

theme_bw( ) +

\#theme (axis.text.x $=$ element_text $($ angle $=90$, hjust $=1$, vjust $=0.5))+$ theme(legend.title=element_blank()) +

scale_color_manual(values=c("\#FF6600", "\#FFCC33", "\#000066")) +

$\operatorname{labs}(\mathrm{x}=$ "aSN expression interval (CRPKMs)", $\mathrm{y}=$ "mean PSI")

theme(axis.text.x $=$ element_text $($ angle $=90$, hjust $=1$, vjust $=0.5)$ )

Event_mean_int_PMCA2 \% \%

$\operatorname{ggp} \bar{l}$ ot ()$^{+}+$

geom_point(mapping $=$ aes $(x=$ asn_exp_interval, $y=$ mean_PSI, color $=$ Eve ntID), size $=2.5)+$

\#geom_errorbar(aes ( $x=a s n \_e x p \_i n t e r v a l, y m i n=m e a n \_P S I-s d \_P S I, y m a x=m e a n \_P$ 


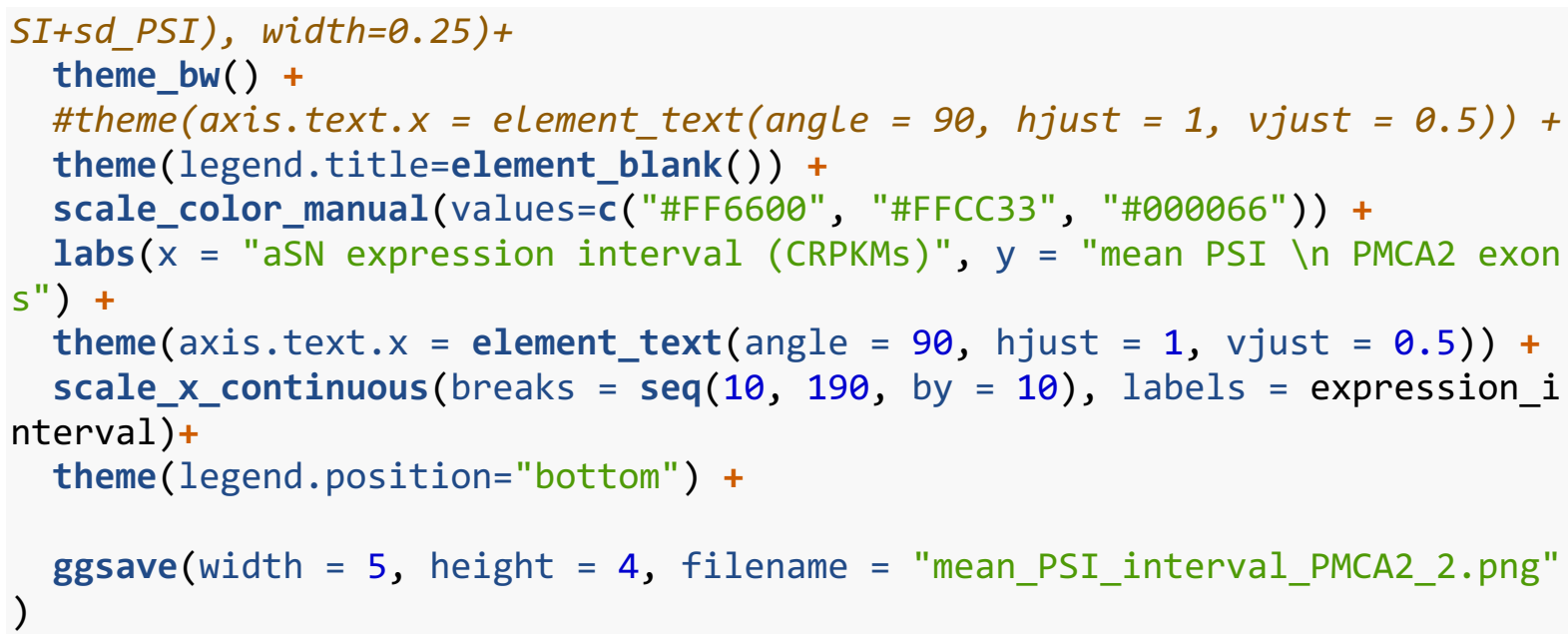

\#t-test - calculate if there is a stastically significant difference betwe en the mean PSI for tissues with aSN expression below 50 CRPKMs and above 50 CRPKMS.

interval_means <- function(PMCA_data, interval1, interval2) \{ exsp_asn <- filter(asn_CRPKMs_mean, CRPKMs >= interval1 \& CRPKMs < interva 12) $\%$

Event_mean_PMCA <- subset(PMCA_data, Subgroup \%in\% exsp_asn\$Subgroup) \%>



tsum.test (mean.x $=$ (low_data_means $[[1,2]]), \mathrm{s} \cdot \mathrm{x}=$ (low_data_means $[[1,3]])$, $\mathrm{n} \cdot \mathrm{x}=($ low_data_means $[[\overline{1}, 4]])$, mean.y $=($ high_data_means $[[1, \overline{1}]]), \mathrm{s} \cdot \mathrm{y}=(\mathrm{h}$ igh_data_means $[\overline{[}[1,3]]), \mathrm{n} \cdot \mathrm{y}=($ high_data_means $[[1, \overline{4}]]))$

\#\#

\#\# Welch Modified Two-Sample t-Test

\#\#

\#\# data: Summarized $\mathrm{x}$ and $\mathrm{y}$

$\# \#=4.9465, \mathrm{df}=18.681, \mathrm{p}$-value $=9.394 \mathrm{e}-05$

\#\# alternative hypothesis: true difference in means is not equal to $\theta$ \#\# 95 percent confidence interval:

\#\# 22.9693956 .73360

\#\# sample estimates: 


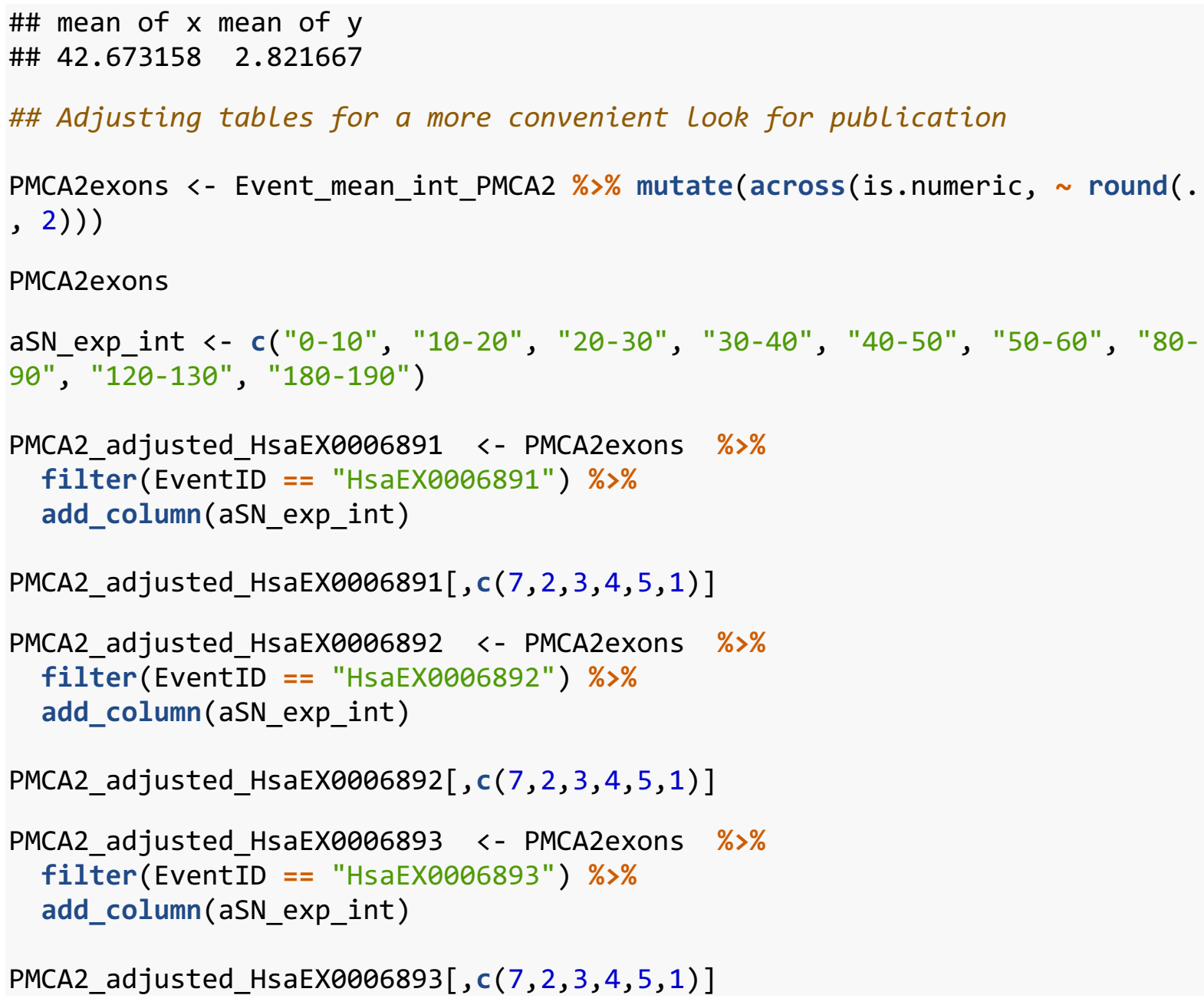

